\title{
The Classical Field Limit of Scattering Theory for Non-Relativistic Many-Boson Systems. II
}

\author{
J. Ginibre ${ }^{1}$ and G. Velo ${ }^{2}$ \\ ${ }^{1}$ Laboratoire de Physique Théorique et Hautes Energies, Université de Paris-Sud, F-91405 Orsay, \\ France \\ ${ }^{2}$ Istituto di Fisica A. Righi, Università di Bologna, and INFN, Sezione di Bologna, I-Bologna, Italy
}

\begin{abstract}
We study the classical field limit of non relativistic many-boson theories in space dimension $n \geqq 3$, extending the results of a previous paper to more singular interactions. We prove the expected results: when $\hbar$ tends to zero, the quantum theory tends in a suitable sense to the corresponding classical field theory, and the quantum fluctuations are governed by the equation obtained by linearizing the quantum evolution equation around the classical solution. These results hold uniformly in time and therefore apply to scattering theory. The interactions considered here are so singular as to require a change of domain in order to define the generator of the evolution of the fluctuations, but sufficiently regular so that no energy renormalization is needed.
\end{abstract}

\section{Introduction}

This is the second paper in which we study the classical field limit of nonrelativistic many-boson theories with two-body interactions in space dimension $n \geqq 3$. A mathematical treatment of this problem has been given previously by Hepp [5] for suitably regular potentials and in finite time intervals. In [2] we have extended Hepp's results to more singular potentials and to the case of infinite time intervals (scattering theory). We refer to the introduction of [2] for a more detailed exposition of the problem and of the results. They consist in proving that, when $\hbar \rightarrow 0$, the difference between the Heisenberg field operators and the solution of the classical field equation is $O\left(\hbar^{1 / 2}\right)$ and evolves according to the equation obtained by linearizing the quantum evolution equation around the classical solution. In a suitable representation of the field operators the estimates are uniform in time and imply the convergence, when $\hbar \rightarrow 0$, of suitable matrix elements of the wave operators and of the $S$-matrix.

Various degrees of difficulty arise in the problem, depending on the regularity of the interaction potential $V$. The role of this regularity is best understood from its bearing on the definition and properties of the unitary group of operators $U_{2}(t, s)$ 
describing the evolution of the quantum fluctuations around the classical limiting solution. In [2] we have treated the most regular case, namely when the generator $H_{2}(t)$ of $U_{2}(t, s)$ has the same domain as $H_{0}+N$ where $H_{0}$ is the free hamiltonian and $N$ is the particle number operator. This corresponds to potentials $V \in L_{\text {loc }}^{2}$, as far as local regularity is concerned. The present paper is devoted to the study of the next level of singularity, where a change of domain is necessary but where $\mathrm{H}_{2}(t)$ still has the same form domain as $H_{0}+N$ and no energy renormalization is needed. This corresponds to potentials $V \in L_{\mathrm{loc}}^{2 n /(n+2)}$. For more singular interactions an infinite energy renormalization for the fluctuations and possibly a change of Hilbert space will be necessary. The situation is somewhat analogous to that encountered in relativistic quantum field theory [4].

In order to implement our program we need to control both the classical and quantum theories. The classical theory is studied in [3] and the relevant results are summarized in Sect. 2 of [2]. Our treatment requires the local regularity condition $V \in L_{\text {loc }}^{n / 4}$, so that the greater generality achieved in this paper as compared to [2] takes place for $3 \leqq n \leqq 7$. In particular for these values of $n$ our analysis covers the case of a potential $V=C|x|^{-\gamma}$ with $C>0$ and $2<\gamma<1+n / 2$.

In order to derive the necessary estimates for the more singular potentials considered here we need stronger regularity properties of the classical solutions than in [2]. In addition we need a boundedness property of some operators uniformly in $\hbar$ and in time (see Condition 2.1). Under a suitable stability assumption on the potential $V$ [see (2.46)], this property can be proved in bounded time intervals. However for infinite time intervals it can only be reduced to time decay estimates of the classical solutions which are likely to hold for smooth dispersive solutions, but which we are unable to prove from first principles. In the derivation of the main results we shall therefore keep Condition 2.1 as a technical assumption.

This paper relies heavily on [2] and is not supposed to be readable by itself. In particular we shall freely use the notation and results of [2]. Proofs will be shortened or even omitted whenever similar to those in [2].

The paper is organized as follows. In Sect. 2 we study the evolution operators both for the quantum theory and for the quantum fluctuations around the classical solution. In Sect. 3 we proceed to the proof of the announced convergence as $\hbar \rightarrow 0$. The main results are stated in Proposition 3.1 for finite time intervals and in Theorem 3.1 for infinite time intervals.

\section{The Quantum Theory and the Quantum Fluctuations}

In this section, we derive the properties of the quantum evolution operators $W(t, s)$ and $\tilde{W}(t, s)$ defined by (1.25) and (1.45) of [2] and of the operators $U_{2}(t, s)$ and $\tilde{U}_{2}(t, s)$, defined by (1.22) and (1.41) of [2], which describe the evolution of the quantum fluctuations. To a large extent we follow Sects. 3 and 4 of [2], with which most definitions and notation are common. In particular, we denote by $|\cdot|_{q}$ the norm in $L^{q} \equiv L^{q}\left(\mathbb{R}^{n}\right)$ except for $q=2$ where the subscript 2 is omitted, by $\mathscr{H}$ the boson Fock space, by $\mathscr{H}_{N}$ the $N$-particle subspace (in particular $\mathscr{H}_{1}=L^{2}$ ), by $\|\Phi\|$ the norm of $\Phi$ in $\mathscr{H}$ and by $\||A|\|$ (resp. $\||A|\|_{N}$ ) the norm of a bounded operator $A$ in $\mathscr{H}$ (resp. in $\mathscr{H}_{N}$ ). If $A$ is semi-bounded, we denote by $Q(A)$ its form domain and 
by $Q^{*}(A)$ the dual of $Q(A)$ where the duality is defined by the scalar product in $\mathscr{H}$. Throughout this section, we omit the dependence of the various operators on the canonical operators $a$.

The Weyl operators are defined by (3.5) of [2] and satisfy Lemma 3.1 of [2]. The evolution operator $U(t, s) \equiv U(t-s)$ is defined by (3.14) of [2] for potentials $V$ satisfying (3.12) of [2]. The operators $H_{k}(k=2,3,4)$ are defined by (3.15)-(3.24) of [2] for $k=2,3$ and by (1.63) of [2] for $k=4$.

We recall that if $n \geqq 3$, if $V \in L^{n / 2}+L^{\infty}$ and if $v$ is the operator of multiplication by $V$ in $\mathscr{H}_{1}$, then for any $\varepsilon>0$, there exists $\bar{b}_{\varepsilon}>0$ such that the following inequality holds in the sense of quadratic forms on $\mathscr{H}_{1} \cap Q\left(H_{0}\right)\left(\equiv H^{1}\right)$ :

$$
|v| \leqq-\varepsilon \Delta+\bar{b}_{\varepsilon} .
$$

From this it follows that

$$
\Gamma_{2}(|V|) \leqq \sum_{i<j}\left(\frac{\varepsilon}{4}\left(k_{i}-k_{j}\right)^{2}+\bar{b}_{\varepsilon}\right),
$$

where $\Gamma_{2}$ is defined by (5.2) of [2] and $k_{i}$ is the momentum of the $i$-th particle,

$$
\begin{aligned}
\ldots & \leqq \varepsilon\left\{\sum_{i<j} \frac{1}{4}\left(k_{i}-k_{j}\right)^{2}+\frac{1}{4}\left(\sum_{i} k_{i}\right)^{2}\right\}+\frac{1}{2} \bar{b}_{\varepsilon} N(N-1) \\
& \leqq \frac{1}{2} N\left(\varepsilon H_{0}+\bar{b}_{\varepsilon} N\right) .
\end{aligned}
$$

In all this section, we assume that $V$ satisfies the condition:

$$
V \in L^{p_{1}}+L^{p_{2}} \text { with } 2 n /(n+2) \leqq p_{2} \leqq p_{1} \leqq \infty .
$$

We recall that for any $q, 1 \leqq q \leqq \infty$, the conjugate index $\bar{q}$ is defined by $1 / q+1 / \bar{q}$ $=1$.

We first derive some properties of $\mathrm{H}_{2}(t)$. In the following three lemmas (Lemmas 2.1 and 2.2 and Corollary 2.1), the time dependence of $\varphi$ and of the various operators is omitted.

Lemma 2.1. Let $V$ satisfy (2.3), $V=V_{1}+V_{2}$ with $V_{i} \in L^{p_{1}}$, and let $\varphi \in L^{2} \cap L^{2 \bar{p}_{2}}$. Then 1) $N^{-1} G$ and $N^{-1} K$ are bounded and satisfy

$$
\begin{aligned}
& \left\|\left|N^{-1} G\right|\right\| \leqq\|g\|_{\infty} \leqq c_{0}, \\
& \left\|\mid N^{-1} K\right\|\|\leqq\| k \|_{1} \leqq c_{0},
\end{aligned}
$$

where

$$
c_{0}=\sum_{i}\left\|V_{i}\right\|_{p_{\imath}}\|\varphi\|_{2 \bar{p}_{i}}^{2}
$$

2) Corresponding to the decomposition $V=V_{1}+V_{2}$, let $L=L_{1}+L_{2}$. Let $0 \leqq \alpha_{i}$ $\leqq \operatorname{Min}\left(p_{i}-1,1\right)$ for $i=1,2$, and define $q_{i}$ by

$$
2 / q_{i}+\left(1+\alpha_{i}\right) / 2 p_{i}=1 \text { for } i=1,2 \text {. }
$$

Then

$$
L_{i}^{*} L_{i} \leqq \frac{1}{2} c_{i}^{2} \Gamma_{2}\left(\left|V_{i}\right|^{1-\alpha_{2}}\right) \quad \text { for } \quad i=1,2,
$$


where

$$
c_{i} \equiv\left\{\int|\varphi|^{2}\left(\left|V_{i}\right|^{1+\alpha_{i}} *|\varphi|^{2}\right)\right\}^{1 / 2} \leqq\left\|V_{i}\right\|_{p_{i}}^{\left(1+\alpha_{i}\right) / 2}\|\varphi\|_{q_{i}}^{2} .
$$

3) Let in addition $\left(1-\alpha_{i}\right) n / 2 \leqq p_{i}$ for $i=1,2$. For any $\varepsilon>0$, let $\bar{b}_{\varepsilon}$ be such that

$$
\left|v_{i}\right|^{1-\alpha_{i}} \leqq-\varepsilon \Delta+\bar{b}_{\varepsilon} \text { for } i=1,2 \text {. }
$$

Let $\omega \in \mathbb{C},|\omega|=1$. Then

$$
\omega L+\bar{\omega} L^{*} \leqq \frac{1}{2}\left(c_{1}+c_{2}\right)\left(\varepsilon H_{0}+\left(\bar{b}_{\varepsilon}+1\right) N+2\right) .
$$

Proof. 1) The first inequalities in (2.4) and (2.5) are obvious, while the last ones with (2.6) follow from Hölder's and Young's inequalities.

2) Let $\Psi$ in $\mathscr{H}$ have finitely many particles and smooth wave functions. Let $X=\left(x_{1}, \ldots, x_{N}\right)$. Then for $0 \leqq \alpha_{i} \leqq 1$,

$$
\begin{aligned}
\left\|L_{i} \Psi\right\|^{2}= & f d X\left|\frac{1}{2} \int d y_{1} d y_{2} \bar{\varphi}\left(y_{2}\right) \bar{\varphi}\left(y_{2}\right) V_{i}\left(y_{1}-y_{2}\right) \Psi\left(X, y_{1}, y_{2}\right)\right|^{2} \\
& \leqq \frac{1}{2} c_{i}^{2} f d X \frac{1}{2} \int d y_{1} d y_{2}\left|V_{i}\left(y_{1}-y_{2}\right)\right|^{1-\alpha_{2}}\left|\Psi\left(X, y_{1}, y_{2}\right)\right|^{2}
\end{aligned}
$$

by Schwarz's inequality,

$$
\ldots=\frac{1}{2} c_{i}^{2}\left\langle\Psi, \Gamma_{2}\left(\left|V_{i}\right|^{1-\alpha_{i}}\right) \Psi\right\rangle .
$$

For $1+\alpha_{i} \leqq p_{i}$ the quantity $c_{i}$ is estimated as in (2.9) by the use of Hölder's and Young's inequalities. Note that $q_{i}(i=1,2)$ lies in the allowed interval $\left[2,2 \bar{p}_{2}\right]$.

3) Note first that the condition $p_{1} \geqq p_{2} \geqq 2 n /(n+2)$ ensures that the conditions $1+\alpha_{i} \leqq p_{i}$ and $\left(1-\alpha_{i}\right) n / 2 \leqq p_{i}$ are compatible, while the latter ensures that $\left|V_{i}\right|^{1-\alpha_{i}} \in L^{n / 2}+L^{\infty}$ and therefore that for any $\varepsilon>0$, there exists $\bar{b}_{\varepsilon}$ such that $(2.10)$ holds. It then follows from $(2.2,8)$ that

$$
L_{i}^{*} L_{i} \leqq \frac{1}{4} c_{i}^{2} N\left(\varepsilon H_{0}+\bar{b}_{\varepsilon} N\right) .
$$

From the inequality

$$
\begin{aligned}
& {\left[\left(\frac{c_{i}}{2}(N+2)\right)^{1 / 2}-\bar{\omega}\left(\frac{c_{i}}{2} N\right)^{-1 / 2} L_{i}^{*}\right]} \\
& \cdot\left[\left(\frac{c_{i}}{2}(N+2)\right)^{1 / 2}-\omega L\left(\frac{c_{i}}{2} N\right)^{-1 / 2}\right] \geqq 0
\end{aligned}
$$

we then obtain

$$
\begin{aligned}
& \omega L_{i}+\bar{\omega} L_{i}^{*} \leqq \frac{1}{2} c_{i}(N+2)+\left(\frac{1}{2} c_{i} N\right)^{-1} L_{i}^{*} L_{i} \\
& \quad \leqq \frac{1}{2} c_{i}\left(\varepsilon H_{0}+\left(\bar{b}_{\varepsilon}+1\right) N+2\right)
\end{aligned}
$$

from which (2.11) follows immediately. Q.E.D.

Lemma 2.1 has immediate consequences for the operator $\mathrm{H}_{2}$.

Corollary 2.1. Let $V$ satisfy (2.3) and $\varphi \in L^{2} \cap L^{2 \bar{p}_{2}}$. Then the operator $\mathrm{H}_{2}$ defined by (3.15)-(3.21) of [2] is bounded from $Q\left(H_{0}+N\right)$ to $Q^{*}\left(H_{0}+N\right)$ and norm continuous as a function of $\varphi$ in $L^{2} \cap L^{2 \bar{p}_{2}}$. Furthermore, there exists a constant $c_{3} \geqq 1$ depending on $V$ but uniform in $\varphi$ for $\varphi$ in a bounded set of $L^{2} \cap L^{2 \bar{p}_{2}}$ such that

$$
\frac{1}{2}\left(H_{0}+c_{3}(N+1)\right) \leqq H_{2}+c_{3}(N+1) \leqq \frac{3}{2}\left(H_{0}+c_{3}(N+1)\right) .
$$


Proof. We prove only the inequality (2.16). By (2.11), there exists a constant $\bar{b}$ depending on $V$ but uniform in $\varphi$ as stated above such that

$$
\pm\left(L+L^{*}\right) \leqq \frac{1}{2} H_{0}+\bar{b}(N+1) \text {. }
$$

The estimates (2.4), (2.5), and (2.17) imply

$$
\frac{1}{2} H_{0}-\left(\bar{b}+2 c_{0}\right)(N+1) \leqq H_{2} \leqq \frac{3}{2} H_{0}+\left(\bar{b}+2 c_{0}\right)(N+1)
$$

from which (2.16) follows after choosing $c_{3}$ such that $c_{3} \geqq \operatorname{Max}\left(1,2 \bar{b}+4 c_{0}\right)$. Q.E.D.

For the estimates of the next section, we shall make essential use of the regularization operator $P_{v \kappa}$ defined in Sect. 3 of [2]. $P_{v \kappa}$ is bounded from $Q^{*}\left(H_{0}\right)$ to $\mathscr{H}$ (because $|k| \hat{\varrho}(k)$ is bounded) and satisfies the following property:

Lemma 2.2. Let $V$ satisfy (2.3) and let $\varphi \in L^{2}$. Then $P_{v \kappa} H_{3}$ and $P_{v \kappa} H_{4}$ are bounded operators from $Q\left(H_{0}\right)$ to $\mathscr{H}$, and the former is norm continuous as a function of $\varphi \in L^{2}$.

Proof. We first prove boundedness. Because of the particle number cut off, it is sufficient to prove that $R_{\kappa} A_{3}, R_{\kappa} A_{3}^{*}$ and $R_{\kappa} H_{4}$ are bounded from $\mathscr{H}_{2} \cap Q\left(H_{0}\right)$, $\mathscr{H}_{1} \cap Q\left(H_{0}\right)$ and $\mathscr{H}_{2} \cap Q\left(H_{0}\right)$ respectively to $\mathscr{H}$. One then sees easily that it is sufficient to prove that the one particle operator $\varrho_{*} V_{y}$ is bounded from $H^{1}$ to $L^{2}$, where $V_{y}$ is the operator of multiplication by the function $V_{y}(x)=V(x-y)$ uniformly with respect to $y$. We factor $\varrho_{*} V_{y}$ as follows :

$$
\varrho_{*} V_{y}=\left\{\varrho_{*}\left|V_{y}\right|^{n /(n+2)}\right\}\left\{V_{y}\left|V_{y}\right|^{-n /(n+2)}\right\} .
$$

Now

$$
|V|^{n /(n+2)} \in L^{2}+L^{\infty}
$$

and

$$
V|V|^{-n /(n+2)} \in L^{n}+L^{\infty} .
$$

Since $\varrho \in L^{1} \cap L^{2}$, the first factor in the R.H.S. of (2.19) is bounded in $L^{2}$ by Hölder's and Young's inequalities, while the second factor is bounded from $H^{1}$ to $L^{2}$ by Hölder's and Sobolev's inequalities, or equivalently by (2.1). Uniformity in $y$ is immediate. This proves boundedness. Continuity follows from the fact that $A_{3}^{*}$ is linear in $\varphi$. Q.E.D.

We are now in a position to prove a regularized version of the differentiability of $\tilde{W}(t, s)$, which is defined as in Sect. 3 of [2].

Proposition 2.1. Let $V$ satisfy (2.3) and $V_{-} \in L^{\operatorname{Max}\left(p_{1}, n / 2\right)}+L^{n / 2}$, let $\varphi \in \mathscr{C}\left(\mathbb{R}, H^{1} \cap L^{2 \bar{p}_{2}}\right)$ and satisfy the Eq. (2.9) of [2]. Then for all vectors $\Psi \in \mathscr{H}$ such that $\tilde{W}(t, s) \Psi \in \mathscr{C}(R$, $Q\left(H_{0}\right)$ ) as a function of $t$ for fixed s (of which there exists a dense set), $P_{v \kappa} \tilde{W}(t, s) \Psi$ is differentiable in $\mathscr{H}$ with derivative given by

$$
\begin{aligned}
i \frac{d}{d t} P_{v \kappa} \tilde{W}(t, s) \Psi= & P_{v \kappa}\left(\tilde{H}_{2}(t)-H_{0}+\hbar^{1 / 2} \tilde{H}_{3}(t)+\hbar \tilde{H}_{4}(t)\right) \\
& \cdot \tilde{W}(t, s) \Psi .
\end{aligned}
$$


Proof. For $\Psi$ in the dense set $\mathscr{D}$ defined by (3.46) of [2], $\tilde{W}(t, s) \Psi \in \mathscr{C}\left(\mathbb{R}, Q\left(H_{0}\right)\right)$. For such $\Psi$, the proof is identical with that of Proposition 3.1 of [2]. Therefore for $\Psi \in \mathscr{D}$

$$
P_{v \kappa}\left(\tilde{W}\left(t^{\prime}, s\right)-\tilde{W}(t, s)\right) \Psi=-i \int_{t}^{t^{\prime}} d \tau P_{v \kappa} \tilde{B}(\tau) \tilde{W}(\tau, s) \Psi
$$

where

$$
\tilde{B}(\tau)=\tilde{H}_{2}(\tau)-H_{0}+\hbar^{1 / 2} \tilde{H}_{3}(\tau)+\hbar \tilde{H}_{4}(\tau) .
$$

By Corollary 2.1 and Lemma 2.2, $P_{v \kappa} \tilde{B}(\tau)$ is bounded and norm continuous in $\tau$ from $Q\left(H_{0}\right)$ to $\mathscr{H}$.

We now take $\Psi$ such that $\tilde{W}(t, s) \Psi \in \mathscr{C}\left(\mathbb{R}, Q\left(H_{0}\right)\right)$ and prove (2.21) for this $\Psi$ by a limiting procedure. Since $U_{0}(s)$ and $C\left(\varphi_{\hbar}(s)\right)$ are fixed unitary operators and since $U_{0}(t)$ and $C\left(\varphi_{\hbar}(t)\right)$ are bounded and strongly continuous in $t$ as operators in $Q\left(H_{0}\right)$, it is sufficient to show that any $\Phi \in \mathscr{H}$ such that $U(t) \Phi \in \mathscr{C}\left(\mathbb{R}, Q\left(H_{0}\right)\right)$ can be approximated by a sequence $\Phi_{n} \in \mathscr{D}(H) \cap \mathscr{C}_{0}(N)$ in the sense that $U(t) \Phi_{n}$ tends to $U(t) \Phi$ in $Q\left(H_{0}\right)$ uniformly in compact intervals. Since $N$ commutes with both $H_{0}$ and $H$, one can first approximate $\Phi$ in this sense by vectors in $\mathscr{C}_{0}(N)$ and therefore restrict one's attention to the case where $\Phi \in \mathscr{C}_{0}(N)$. For such a $\Phi$, one can take

$$
\Phi_{n}=n \int_{0}^{1 / n} d \tau U(\tau) \Phi
$$

as a strong Riemann integral in $Q\left(H_{0}\right)$. Then

$$
U(t)\left(\Phi_{n}-\Phi\right)=n \int_{0}^{1 / n} d \tau(U(t+\tau)-U(t)) \Phi
$$

and therefore

$$
\left.\left\|U(t)\left(\Phi_{n}-\Phi\right)\right\|_{Q\left(H_{0}\right)} \leqq \operatorname{Sup}_{0 \leqq} \| U(t+\tau)-U(t)\right) \Phi \|_{Q\left(H_{0}\right)} .
$$

Since $U(t) \Phi \in \mathscr{C}\left(\mathbb{R}, Q\left(H_{0}\right)\right)$, it is uniformly continuous on compact intervals. This proves the required convergence, and therefore (2.21) for all $\Psi$ such that $\tilde{W}(t, s) \Psi \in \mathscr{C}\left(\mathbb{R}, Q\left(H_{0}\right)\right)$. Differentiability follows from (2.21). Q.E.D.

In order to prove our main result in Sect. 3, we shall need an additional property of $\tilde{W}(t, s)$ which we state in the form of the following condition.

Condition 2.1. 1) There exists a fixed (i.e., independent of $t, s$, and $\hbar$ ) dense set $\mathscr{D}_{1}$ in $\mathscr{H}$ such that for any $\Psi \in \mathscr{D}_{1}$ for any $s \in \mathbb{R}, \tilde{W}(\cdot, s) \Psi \in \mathscr{C}\left(\mathbb{R}, Q\left(H_{0}+N\right)\right)$ and $\tilde{W}(t, s) \Psi$ is bounded in $Q\left(H_{0}+N\right)$ uniformly with respect to $\hbar$ for $0<\hbar \leqq \hbar_{0}$ for some $\hbar_{0}$ depending only on $V$, and uniformly with respect to $t, s$ in compact intervals.

2) In addition, for any $\Psi \in \mathscr{D}_{1}, \tilde{W}(t, s) \Psi$ is bounded in $Q\left(H_{0}+N\right)$ uniformly with respect to $\hbar$ for $0<\hbar \leqq \hbar_{0}$ and uniformly with respect to $t, s$ in $\mathbb{R}^{+}$(resp. $\mathbb{R}^{-}$, resp. $\mathbb{R})$.

[The uniformity in $t, s$ is relevant to ensure the uniformity in $t, s$ of the $\hbar \rightarrow 0$ limit in the corresponding ranges of $t, s$ and the existence of the $\hbar \rightarrow 0$ limit for the wave operators $\Omega_{+}$(resp. the wave operator $\Omega_{-}$, resp. the $S$-matrix).] 
Under the additional assumption that the potential be stable in a suitable sense (see Condition 2.2 below), we shall prove part 1) of Condition 2.1. However, we shall not be able to prove part 2) from first principles, and we shall only reduce it to some decay properties of the classical solution $\varphi(t)$. This will be done at the end of this section after the study of the quantum fluctuations, to which we turn now.

In order to define the unitary groups $U_{2}(t, s)$ and $\tilde{U}_{2}(t, s)$ we need to solve the evolution Eqs. (1.22) and (1.42) of [2] with time dependent generators $\mathrm{H}_{2}(t)$ and $\tilde{H}_{2}(t)-H_{0}$. For the regular potentials considered in [2], the perturbation $H_{2}-H_{0}$ was bounded by $N$, and it was therefore simpler to disregard $U_{2}(t, s)$ and consider $\tilde{U}_{2}(t, s)$ directly. Since here $H_{2}-H_{0}$ is only bounded by $H_{0}+N$ (cf. Corollary 2.1), this is no longer the case, and we shall therefore study $U_{2}(t, s)$ first. The existence and relevant properties of $\tilde{U}_{2}(t, s)$ will follow immediately from those of $U_{2}(t, s)$. We shall make extensive use of the spaces $\mathscr{H}^{\delta}$ defined for $-1 \leqq \delta \leqq 1$ by $\mathscr{H}^{\delta}=Q\left(\left(H_{0}+N\right)^{\delta}\right)$ if $\delta \geqq 0$ and by $\mathscr{H}^{\delta}=Q^{*}\left(\left(H_{0}+N\right)^{|\delta|}\right)$ if $\delta \leqq 0$. Note that these spaces are different from those with the same name used in [2].

Proposition 2.2. Let $V$ satisfy (2.3) and let $\varphi \in \mathscr{C}^{1}\left(\mathbb{R}, L^{2} \cap L^{2 \bar{p}_{2}}\right)$. Then there exists a unique group of operators $U_{2}(t, s)$ satisfying the following properties:

1) For each $\delta \in[-1,+1], U_{2}(t, s)$ is bounded and strongly continuous with respect to $t, s$ in $\mathscr{H}^{\delta}$.

2) $U_{2}(t, s)$ is unitary in $\mathscr{H}$.

3) $U_{2}(t, s)$ is strongly differentiable from $\mathscr{H}^{1}$ to $\mathscr{H}^{-1}$ and

$$
i \frac{d}{d t} U_{2}(t, s)=H_{2}(t) U_{2}(t, s) \text {. }
$$

Proof. This is a special case of a general result of Kato [6], (Theorems 4.1, 5.1, 5.2, and Remarks 5.3 and 5.4), where we take $X=\mathscr{H}^{-1}$ and $Y=\mathscr{H}^{1}$. The proof is greatly simplified by the fact that we deal with a scale of Hilbert spaces, and we restrict our attention to the verification of the main assumptions. That $\mathscr{H}^{1}$ and $\mathscr{H}^{-1}$ are $H_{2}(t)$-admissible for each $t$ and that $H_{2}(t)$ is norm continuous in $t$ from $\mathscr{H}^{1}$ to $\mathscr{H}^{-1}$ follows from Corollary 2.1 , from the estimate $(2.26)$ below and from Proposition 2 of [7].

There remains to be proved that $H_{2}(t)$ is stable in $\mathscr{H}^{1}$ and/or in $\mathscr{H}^{-1}$ (one follows from the other by duality), or equivalently that the Kato approximants with stepwise constant generator are uniformly bounded in $\mathscr{H}^{1}$ and/or in $\mathscr{H}^{-1}$ for $t, s$ in a compact interval. Let therefore $I$ be a compact interval and choose the constant $c_{3}$ such that (2.16) holds for all $t \in I$. Let $A_{2}(t)=H_{2}(t)+c_{3}(N+1)$ and define the family of equivalent norms in $\mathscr{H}^{-1}$

$$
\|\Phi\|_{t}=\left\|A_{2}(t)^{-1 / 2} \Phi\right\| .
$$

From (2.11) with $\varepsilon=1$ and from (2.16), it follows that

$$
\begin{aligned}
& \pm i\left[H_{2}(t), A_{2}(t)\right]= \pm 2 i c_{3}\left(L(t)-L^{*}(t)\right) \\
& \quad \leqq c_{3}\left(c_{1}(t)+c_{2}(t)\right)\left(H_{0}+\left(\bar{b}_{1}+1\right) N+2\right) \\
& \quad \leqq 2 c_{4}(t) A_{2}(t),
\end{aligned}
$$


where

$$
c_{4}(t)=\left(c_{3}+\vec{b}_{1}+1\right)\left(c_{1}(t)+c_{2}(t)\right)
$$

and $c_{i}(t), i=1,2$, are defined and estimated by (2.9).

Furthermore, from estimates similar to those in Lemma 2.1, it follows that $A_{2}(t)$ is norm differentiable from $\mathscr{H}^{1}$ to $\mathscr{H}^{-1}$ and that its time derivative satisfies the estimate

$$
\pm \dot{A}_{2}(t) \leqq 4 c_{0}^{\prime}(t) N+\left(c_{1}^{\prime}(t)+c_{2}^{\prime}(t)\right)\left(\varepsilon H_{0}+\left(\bar{b}_{\varepsilon}+1\right) N+2\right),
$$

where

$$
\begin{aligned}
& c_{0}^{\prime}(t)=\sum_{i=1,2}\left\|V_{i}\right\|_{p_{l}}\|\varphi\|_{2 \bar{p}_{l}}\|\dot{\varphi}\|_{2 \bar{p}_{l}}, \\
& c_{i}^{\prime}(t)=\left\|V_{i}\right\|_{p_{l}}^{\left(1+\alpha_{\imath}\right) / 2}\|\varphi\|_{q_{l}}\|\dot{\varphi}\|_{q_{l}} \text { for } \quad i=1,2,
\end{aligned}
$$

where $q_{i}$ is defined by (2.7) and $\dot{\varphi}$ is the time derivative of $\varphi$. From (2.28) with $\varepsilon=1$ and (2.16), we obtain

$$
\pm \dot{A}_{2}(t) \leqq 2 c_{5}(t) A_{2}(t)
$$

where

$$
c_{5}(t)=4 c_{0}^{\prime}(t)+\left(\widetilde{b}_{1}+2\right)\left(c_{1}^{\prime}(t)+c_{2}^{\prime}(t)\right)
$$

and we have used the fact that $c_{3} \geqq 1$. Using (2.26) and (2.31), one easily shows by differentiation and integration that for all $\Phi \in \mathscr{H}^{-1}$ and all $s, t \in I$,

$$
\begin{aligned}
& \left\|\exp \left(i \tau H_{2}(t)\right) \Phi\right\|_{t} \leqq \exp \left(|\tau| c_{4}(t)\right)\|\Phi\|_{t}, \\
& \|\Phi\|_{t} \leqq \exp \left(\mid \int_{s}^{t} d \tau c_{5}(\tau)\right)\|\Phi\|_{s} .
\end{aligned}
$$

By Proposition 3.4 of [6], these estimates imply that $H_{2}(t)$ is stable in $\mathscr{H}^{-1}$. Stability in $\mathscr{H}^{1}$ follows by duality. Q.E.D.

As a by-product of the proof of Proposition 2.2, we obtain norm estimates for $U_{2}(t, s)$ in $\mathscr{H}^{ \pm 1}$.

Corollary 2.2. Let $V$ and $\varphi$ satisfy the assumptions of Proposition 2.2 and choose $c_{3}$ such that (2.16) holds for all $t$ in some interval $I$ of $\mathbb{R}$. Then

1) For any $\Phi$ in $\mathscr{H}^{-1}$, for any $t, s$ in $I$,

$$
\left\|U_{2}(t, s) \Phi\right\|_{t} \leqq \exp \left(\left|\int_{s}^{t} d \tau \bar{c}(\tau)\right|\right)\|\Phi\|_{s},
$$

where

$$
\bar{c}(\tau)=c_{4}(\tau)+c_{5}(\tau)
$$

2) Define the norms

$$
\|\Phi\|_{ \pm}=\left\|\left(H_{0}+N+1\right)^{ \pm 1 / 2} \Phi\right\|
$$


in $\mathscr{H}^{ \pm 1}$ and denote by $\|\cdot \mid\|_{ \pm}$the corresponding operator norms in $\mathscr{H}^{ \pm 1}$. Then for all $t, s$ in $I$

$$
\left\|\left|U_{2}(t, s)\right|\right\|_{ \pm} \leqq\left(3 c_{3}\right)^{1 / 2} \exp \left(\left|\int_{s}^{t} d \tau \bar{c}(\tau)\right|\right) .
$$

Since $U_{0}(t)$ is a unitary group both in $\mathscr{H}^{1}$ and $\mathscr{H}^{-1}$, [equipped with the norms (2.37)], the properties of $U_{2}(t, s)$ imply similar properties of $\tilde{U}_{2}(t, s)$

Corollary 2.3. Under the assumptions of Proposition 2.2, $\tilde{U}_{2}(t, s)$ satisfies all the properties stated for $U_{2}(t, s)$ in that proposition, with however (2.23) replaced by

$$
i \frac{d}{d t} \tilde{U}_{2}(t, s)=\left(\tilde{H}_{2}(t)-H_{0}\right) \tilde{U}_{2}(t, s)
$$

Furthermore $\tilde{U}_{2}(t, s)$ satisfies the estimate (cf. (2.38))

$$
\left\|\tilde{U}_{2}(t, s) \mid\right\|_{ \pm} \leqq\left(3 c_{3}\right)^{1 / 2} \exp \left(\left|\int_{s}^{t} d \tau \bar{c}(\tau)\right|\right) .
$$

We next study the behaviour of $\tilde{U}_{2}(t, s)$ as $t$ and/or $s$ tend to $\pm \infty$.

Proposition 2.3. Let $V$ satisfy (2.3), let $\varphi \in \mathscr{C}^{1}\left(\mathbb{R}, L^{2} \cap L^{2 \bar{p}_{2}}\right)$, let $\varphi$ be bounded in $L^{2} \cap L^{2 \bar{p}_{2}}$ uniformly in $t$ for $t \in \mathbb{R}^{+}$(resp. $\mathbb{R}^{-}$, resp. $\mathbb{R}$ ), and let the functions $c_{i}(t)$ $(i=1,2)$ defined by $(2.9)$ and $c_{i}^{\prime}(t)(i=0,1,2)$ defined by (2.29) and (2.30) be integrable at $+\infty$ (resp. at $-\infty$, resp. in $\mathbb{R})$. Then

1) $U_{2}(t, s)$ and $\tilde{U}_{2}(t, s)$ are bounded in $\mathscr{H}{ }^{ \pm 1}$ uniformly in $t, s$ for $t, s$ in $\mathbb{R}^{+}$(resp. in $\mathbb{R}^{-}$, resp. in $\mathbb{R}$ ).

2) Let in addition $c_{0}(t)$ defined by (2.6) be integrable at $+\infty$ (resp. at $-\infty$, resp. in $\mathbb{R}$ ). Then, when $t$ and/or $s$ tend to $+\infty$ (resp. to $-\infty$, resp. to $\pm \infty), \tilde{U}_{2}(t, s)$ has norm limits as an operator from $\mathscr{H}^{1}$ to $\mathscr{H}^{-1}$ and therefore strong limits as an operator in $\mathscr{H}^{\delta}$ for $-1 \leqq \delta \leqq 1$.

Proof. By the boundedness property of $\varphi$ and Corollary 2.1, one can choose the constant $c_{3}$ such that (2.16) holds for all $t$ in $\mathbb{R}^{+}$(resp. $\mathbb{R}^{-}$, resp. $\mathbb{R}$ ). Part 1 ) then follows from (2.38), (2.40), (2.36), (2.27), and (2.32). Part 2) is proved in the same way as part 2) of Proposition 4.2 of [2]. Q.E.D.

We conclude this section by analyzing Condition 2.1 for stable potentials (see below), proving part 1) and reducing part 2) to decay estimates on the classical solution $\varphi$. This requires first some estimates on $H_{3}(t)$ stated in the next lemma (where the time dependence is omitted for brevity).

Lemma 2.3. Let $V$ satisfy (2.3) and let $\varphi \in L^{2} \cap L^{2 \bar{p}_{2}}$. Then $A_{3}$ (defined by (3.24) of [2]) satisfies the estimate

$$
A_{3}^{*} A_{3} \leqq 2 c_{0}(N-1) \Gamma_{2}(|V|),
$$

where $c_{0}$ is defined by (2.6). Furthermore, for any $\omega \in \mathbb{C},|\omega|=1$, and any $\lambda \in \mathbb{R}$,

$$
\hbar^{1 / 2}\left(\omega A_{3}+\bar{\omega} A_{3}^{*}\right) \leqq 2 \lambda c_{0} N+\lambda^{-1} \hbar \Gamma_{2}(|V|) .
$$


Proof. Let $\Phi$ be a vector in $\mathscr{H}$ with finitely many particles and smooth wave functions. Let $X=\left(x_{1}, \ldots, \mathrm{x}_{N}\right)$. Then

$$
\left(A_{3} \Phi\right)(X)=\int d x \sum_{1 \leqq i \leqq N} \bar{\varphi}(x) V\left(x-x_{i}\right) \Phi(X, x)
$$

so that

$$
\begin{aligned}
\left\|A_{3} \Phi\right\|^{2} \leqq & f d X \int d x \sum_{i}\left|V\left(x-x_{i}\right)\right||\Phi(X, x)|^{2} \\
& \cdot N \operatorname{Sup}_{x} \int d y|\varphi(y)|^{2}|V(x-y)| \\
\leqq & c_{0}\left\langle\Phi, 2(N-1) \Gamma_{2}(|V|) \Phi\right\rangle
\end{aligned}
$$

by Schwarz's inequality. This proves (2.41). In order to prove (2.42), we note that for all $\lambda \in \mathbb{R}$,

$$
\left((\lambda N)^{1 / 2}-\bar{\omega}(\lambda(N-1))^{-1 / 2} A_{3}^{*}\right)\left((\lambda N)^{1 / 2}-\omega A_{3}(\lambda(N-1))^{-1 / 2}\right) \geqq 0
$$

and therefore

$$
\omega A_{3}+\bar{\omega} A_{3}^{*} \leqq \lambda N+\lambda^{-1}(N-1)^{-1} A_{3}^{*} A_{3},
$$

from which (2.42) follows by the use of (2.41) and the replacement of $\lambda$ by $2 \lambda c_{0} \hbar^{-1 / 2}$. Q.E.D.

We now restrict our attention to potentials satisfying the following stability condition:

Condition 2.2. (Stability of the potential.) There exist $\eta>0, \beta_{0} \geqq 0$ and $\beta_{1} \geqq 0$ such that the following inequality holds in the sense of quadratic forms

$$
\Gamma_{2}(V-\eta|V|)+\beta_{0} H_{0}+\beta_{1} N \geqq 0 .
$$

This condition is closely related to the stability condition used in Statistical Mechanics ([8], pp. 33-40). In particular, if the modified potential $V-\eta|V|$ obtained from the original one $V$ by slightly reducing its positive part and slightly increasing its negative part is stable in the sense of [8], Condition 2.2 is satisfied (with $\beta_{0}=0$ ). We refer to [8] for the description of a large class of potentials satisfying this condition. Positive potentials obviously satisfy Condition 2.2 (with $\beta_{0}=\beta_{1}=0$ ).

For potentials satisfying Condition 2.2, Lemma 2.3 implies the following result.

Lemma 2.4. Let $V$ satisfy (2.3) and Condition 2.2, let $\hbar \leqq \hbar_{0} \equiv \operatorname{Min}\left(1,\left(4 \beta_{0}\right)^{-1}\right)$, and let $\varphi \in L^{2} \cap L^{2 \bar{p}_{2}}$. Then the following inequalities hold in the sense of quadratic forms

$$
\begin{aligned}
& \frac{1}{3}\left(H_{0}+\hbar H_{4}+c_{8}(N+1)\right) \leqq A_{1}(t) \leqq \frac{5}{3}\left(H_{0}+\hbar H_{4}+c_{8}(N+1)\right), \\
& \frac{1}{4}\left(H_{0}+c_{3}(N+1)\right) \leqq A_{1}(t) \leqq 2\left(H_{0}+\hbar_{0} H_{4}+2 c_{8}(N+1)\right),
\end{aligned}
$$

where $A_{1}(t)$ is defined by

$$
A_{1}(t)=H_{2}(t)+\hbar^{1 / 2} H_{3}(t)+\hbar H_{4}+c_{8}(N+1)
$$


and $c_{8}$ is a constant depending only on $c_{3}$ and on the constants $\eta, \beta_{0}$, and $\beta_{1}$. One can take for instance

$$
c_{8}=\frac{3}{4} c_{3}\left(1+\frac{3}{2 \eta}\right)+\beta_{1} .
$$

Proof. The result follows by an elementary computation from (2.42) with various choices of $\lambda$ and $\omega=\bar{\omega}= \pm 1$, from Condition 2.2, from (2.16) and from the fact that $c_{3} \geqq 4 c_{0}$. Q.E.D.

We can now prove the following result.

Proposition 2.4. Let $V$ satisfy (2.3) and Condition 2.2, let $\hbar_{0}=\operatorname{Min}\left(1,\left(4 \beta_{0}\right)^{-1}\right)$ and let $\varphi \in \mathscr{C}^{1}\left(\mathbb{R}, L^{2} \cap L^{2 \bar{p}_{2}}\right)$. Then

1) $W(t, s)$ is bounded and strongly continuous in $t$ at fixed srom $Q\left(H_{0}+\hbar_{0} H_{4}\right.$ $\left.+2 c_{8}(N+1)\right)$ to $Q\left(H_{0}+N\right)$ and $W(t, s)$ and $\tilde{W}(t, s)$ are bounded and strongly continuous in $t$ for fixed s from $Q\left(\left(N_{0}\right)^{(n+2) / 4}+N^{2}\right)$ to $Q\left(H_{0}+N\right)$. Boundedness is uniform with respect to $\hbar$ for $0<\hbar \leqq \hbar_{0}$ and with respect to $t$ and $s$ in compact intervals.

2) Let in addition $\varphi$ be bounded in $L^{2} \cap L^{2 \bar{p}_{2}}$ uniformly in $t$ for $t \in \mathbb{R}^{+}$(resp. $\mathbb{R}^{-}$, resp. $\mathbb{R}$ ), and let the functions $c_{i}(t), i=1,2$, defined by $(2.9), c_{i}^{\prime}(t), i=0,1,2$, defined by (2.29) and (2.30), $c_{0}(t)^{1 / 2}$ defined by (2.6) and $c_{0}^{\prime \prime}(t)^{1 / 2}$ defined $b y$

$$
c_{0}^{\prime \prime}(t)=\sum_{i}\left\|V_{i}\right\|_{p_{t}}\|\dot{\varphi}\|_{2 \tilde{p}_{t}}^{2}
$$

be integrable at $+\infty$ (resp. at $-\infty$, resp. in $\mathbb{R})$. Then the boundedness properties of 1) hold uniformly for $t, s \in \mathbb{R}^{+}$(resp. $\mathbb{R}^{-}$, resp. $\mathbb{R}$ ).

Note that part 1) [resp. part 2)] of Proposition (2.4) implies part 1) [resp. part 2)] of Condition 2.1 with $\mathscr{D}_{1}=Q\left(\left(N H_{0}\right)^{(n+2) / 4}+N^{2}\right)$.

Proof. We prove only the statements relative to $W(t, s)$, from which those relative to $\tilde{W}(t, s)$ follow immediately. Let $I$ be an interval (possibly unbounded) of the real line and let us choose the constant $c_{8}$ such that (2.47) and (2.48) hold for all $t \in I$. This is always possible if $\varphi(t)$ is bounded in $L^{2} \cap L^{2 \bar{p}_{2}}$ uniformly for $t \in I$. The case where $I$ is bounded (resp. unbounded) is relevant for the proof of part 1) [resp. part 2)]. Let now $\hbar$ be fixed and define $A_{1}(t)$ by (2.49). From (2.11) with $\varepsilon=1$ and (2.42) with $\lambda=c_{0}(t)^{-1 / 2}$ it follows that

$$
\begin{aligned}
\pm & i\left[H_{2}(t)+\hbar^{1 / 2} H_{3}(t)+\hbar H_{4}, A_{1}(t)\right] \\
= & \pm i c_{8}\left(2\left(L(t)-L^{*}(t)\right)+A_{3}(t)-A_{3}^{*}(t)\right) \\
\leqq & c_{8}\left\{\left(c_{1}(t)+c_{2}(t)\right)\left(H_{0}+\left(\bar{b}_{1}+1\right) N+2\right)\right. \\
& \left.+c_{0}(t)^{1 / 2}\left(2 N+\hbar H_{4}\right)\right\} \\
\leqq & 2 c_{6}(t) A_{1}(t) .
\end{aligned}
$$

where

$$
c_{6}(t)=\frac{3}{2}\left(c_{8}+\bar{b}_{1}+2\right)\left(c_{1}(t)+c_{2}(t)+c_{0}(t)^{1 / 2}\right) .
$$


Furthermore $A_{1}(t)$ is norm differentiable from $Q\left(H_{0}+\hbar H_{4}+c_{8}(N+1)\right)$ to $Q^{*}\left(H_{0}\right.$ $\left.+\hbar H_{4}+c_{8}(N+1)\right)$ and its time derivative satisfies the estimate

$$
\begin{aligned}
\pm \dot{A}_{1}(t) \leqq & 4 c_{0}^{\prime}(t) N+\left(c_{1}^{\prime}(t)+c_{2}^{\prime}(t)\right)\left(H_{0}+\left(\bar{b}_{1}+1\right) N+2\right) \\
& +c_{0}^{\prime \prime}(t)^{1 / 2}\left(2 N+\hbar H_{4}\right) \\
\leqq & 2 c_{7}(t) A_{1}(t)
\end{aligned}
$$

where

$$
c_{7}(t)=2\left(c_{5}(t)+2 c_{0}^{\prime \prime}(t)^{1 / 2}\right)
$$

and $c_{5}(t)$ and $c_{0}^{\prime \prime}(t)$ are defined by $(2.32)$ and (2.50) respectively.

Let now $s$ be fixed and define

$$
\mathscr{D}_{2}=\left\{\Psi \in \mathscr{H}: C\left(\varphi_{\hbar}(s)\right) \Psi \in \mathscr{D}(H) \cap \mathscr{D}(N)\right\} \text {. }
$$

By Lemma 3.1 of [2], $W(t, s) \Psi$ is strongly differentiable in $\mathscr{H}$ for all $\Psi$ in $\mathscr{D}_{2}$. For such $\Psi$, one can then estimate by differentiation and integration

$$
\begin{aligned}
\left\|A_{1}(t)^{-1 / 2} W(t, s) \Psi\right\| \leqq & \left\|A_{1}(s)^{-1 / 2} \Psi\right\| \\
& \cdot \exp \left\{\left|\int_{s}^{t} d \tau\left(c_{6}(\tau)+c_{7}(\tau)\right)\right|\right\} .
\end{aligned}
$$

This estimate extends by continuity to all $\Psi$ in $Q^{*}\left(H_{0}+\hbar H_{4}+c_{8}(N+1)\right)$. By duality and use of (2.48), it implies the estimate

$$
\begin{aligned}
& \|\|\left(H_{0}+c_{3}(N+1)\right)^{1 / 2} W(t, s)\left(H_{0}+\hbar_{0} H_{4}+2 c_{8}(N+1)\right)^{-1 / 2} \mid \| \\
& \quad \leqq 2^{3 / 2} \exp \left\{\left|\int_{s}^{t} d \tau\left(c_{6}(\tau)+c_{7}(\tau)\right)\right|\right\}
\end{aligned}
$$

for all $\hbar \in\left(0, \hbar_{0}\right]$ and all $t, s$ in $I$. The boundedness properties of $W(t, s)$ from $Q\left(H_{0}+\hbar_{0} H_{4}+2 c_{8}(N+1)\right)$ to $Q\left(H_{0}+N\right)$ both in parts 1$)$ and 2$)$, follow immediately from the estimate (2.58). Strong continuity follows from weak continuity and the continuity of the norm, which in turns follows from similar estimates. Finally, boundedness and strong continuity of $W(t, s)$ from $Q\left(\left(N H_{0}\right)^{(n+2) / 4}+N^{2}\right)$ to $Q\left(H_{0}+N\right)$ follow from the preceding results and the inequality

$$
H_{4} \leqq \beta_{2}\left(\left(N H_{0}\right)^{(n+2) / 4}+N^{2}\right),
$$

where $\beta_{2}$ is a constant depending only on $V$. (2.59) follows from Sobolev's inequality and elementary estimates. Q.E.D.

The integrability conditions of $c_{i}(t), i=1,2$, and $c_{i}^{\prime}(t), i=0,1,2$, that appear in Proposition 2.4 are the same as in Proposition 2.3. For dispersive classical solutions with suitably regular initial data, they follow from our general theory of the classical equation [3]. This is not the case, however, for the integrability conditions of $c_{0}(t)^{1 / 2}$ and $c_{0}^{\prime \prime}(t)^{1 / 2}$. These conditions are equivalent to the integrability of $\|\varphi(t)\|_{2 \bar{p}_{t}}$ and $\|\dot{\varphi}(t)\|_{2 \bar{p}_{2}}, i=1,2$. For solutions of the free equation with suitable initial data, they are satisfied provided $p_{2} \leqq p_{1}<n / 2$, a condition that we shall require anyway in order to control the $\hbar \rightarrow 0$ limit in Sect. 3. Since dispersive solutions of the classical equation tend to behave asymptotically in time like 
solutions of the free classical equation, it is reasonable to expect these integrability conditions to hold also in the interacting case.

We shall not go further in the reduction of Condition 2.1, and we shall use it as a technical assumption wherever needed in Sect. 3.

\section{The Limit $\hbar \rightarrow 0$}

In this section, we prove the main result of this paper, namely the strong convergence of $\tilde{W}(t, s)$ to $\tilde{U}_{2}(t, s)$ uniformly in $t, s$. We follow to a large extent Sect. 5 of [2] with which most of the notation is common. In all this section, we assume that $V$ and $\varphi$ satisfy the conditions

$$
\begin{aligned}
& \left\{\begin{array}{l}
V \in L^{p_{1}}+L^{p_{2}} \quad \text { with } \quad 2 n /(n+2) \leqq p_{2} \leqq p_{1} \leqq \infty, \\
V_{-} \in L^{\operatorname{Max}\left(p_{1}, n / 2\right)}+L^{n / 2},
\end{array}\right. \\
& \varphi \in \mathscr{C}\left(\mathbb{R}, H^{1} \cap L^{2 \bar{p}_{2}}\right) \cap \mathscr{C}^{1}\left(\mathbb{R}, L^{2} \cap L^{2 \bar{p}_{2}}\right),
\end{aligned}
$$

and that $\varphi$ satisfies the Eq. (2.9) of [2]. We define $b_{1}$ and $b_{2}$ by (5.3) and (5.4) of [2] and $B_{i}=\Gamma_{i}\left(b_{i}\right), i=1,2$ (cf. (5.1) and (5.2) of [2]). In all this section, we assume that part 1) of Condition 2.1 is satisfied and that the set $\mathscr{D}_{1}$ which appears in this condition is such that $\mathscr{D}_{1} \cap Q\left(N B_{1}\right)$ is dense in $\mathscr{H}$. Note that by definition $\mathscr{D}_{1} \subset Q\left(H_{0}+N\right)$. We take a fixed $\Psi \in \mathscr{D}_{1} \cap Q\left(N B_{1}\right)$, we define $\Psi_{i}(t), i=1,2$, by (5.7) of [2], and we start estimating the quantity $\left\|\Psi_{1}(t)-\Psi_{2}(t)\right\|^{2}$, which we decompose as in (5.8)-(5.13) of [2]. We consider the various terms $J_{i}$ of this decomposition successively. As in Sect. 5 of [2], we omit the dependence on $v, \kappa$, most of the time.

Lemma 3.1. 1) $J_{0}$ tends to zero as $v, \kappa \rightarrow \infty$ uniformly for $\Psi$ in a bounded set of $Q\left(H_{0}+N\right)$.

2) $J_{1}$ tends to zero as $v, \kappa \rightarrow \infty$ uniformly for $t, s$ in a compact interval and uniformly for $\Psi$ in a bounded set of $Q\left(H_{0}+N\right)$. If in addition the assumptions of Proposition 2.3, part 1), hold, the convergence is uniform for $t, s$ in $\mathbb{R}^{+}$(resp. $\mathbb{R}^{-}$, resp. $\mathbb{R})$.

Proof. We note that

$$
\begin{aligned}
\mathbb{1}-P & \leqq \mathbb{1}-\sigma+\mathbb{1}-R \leqq \mathbb{1}-\sigma+\Gamma_{1}\left(\mathbb{1}-\varrho_{*}\right) \\
& \leqq\left(v^{-1}+\gamma_{1}\right)\left(H_{0}+N+1\right),
\end{aligned}
$$

where

$$
\gamma_{1}=\operatorname{Sup}_{k}(1-\hat{\varrho}(k))\left(k^{2} / 2+1\right)^{-1} .
$$

Therefore

$$
\begin{aligned}
& J_{0} \leqq\left(v^{-1}+\gamma_{1}\right)\|\Psi\|_{+}^{2}, \\
& J_{1} \leqq\left(v^{-1}+\gamma_{1}\right)^{1 / 2}\|\Psi\|\left\|\Psi_{2}(t)\right\|_{+} .
\end{aligned}
$$

The assumptions made on $\varrho$ ensure that $\gamma_{1} \rightarrow 0$ when $\kappa \rightarrow \infty$. The result then follows from (3.6) and (3.7) and Proposition 2.3, part 1). Q.E.D. 
We now turn to $J_{2}$.

Lemma 3.2. $J_{2}$ satisfies the estimate

$$
\begin{aligned}
J_{2} \leqq & 2 \int_{s}^{t} d \tau\left\{4 \gamma_{2} c_{0}(\tau)+\left(\left(\bar{b}_{1}+2\right) v^{-1}\left\|\sigma_{1}^{\prime}\right\|_{\infty}+\varepsilon^{1 / 2}+2 \varepsilon^{-1 / 2} \bar{b}_{\varepsilon} \gamma_{2}^{2}\right.\right. \\
& \left.\cdot\left(c_{1}(\tau)+c_{2}(\tau)\right)\right\}\left\|\Psi_{1}(\tau)\right\|_{+}\left\|\Psi_{2}(\tau)\right\|_{+}
\end{aligned}
$$

for any $\varepsilon>0$, where $\sigma_{1}$ is the particle number cut off function, $\sigma_{1}^{\prime}$ its derivative, $c_{i}(\cdot)$, $i=0,1,2$, are defined by (2.6) and (2.9), and

$$
\gamma_{2}=\operatorname{Sup}_{k}(1-\varrho(k))\left(k^{2} / 2+1\right)^{-1 / 2} \text {. }
$$

In particular, if the assumptions of Proposition 2.3, part 2), and Condition 2.1 hold, $J_{2}$ tends to zero as $\nu, \kappa \rightarrow \infty$ uniformly for $t, s$ in $\mathbb{R}^{+}$(resp. $\mathbb{R}^{-}$, resp. $\mathbb{R}$ ).

Proof. We observe that

$$
\left[\tilde{H}_{2}(\tau), P\right]=\left[\tilde{H}_{2}(\tau), \sigma\right] R+\sigma\left[\tilde{H}_{2}(\tau), R\right]
$$

and

$$
\left[\tilde{H}_{2}(\tau), \sigma\right]=(\sigma(N+2)-\sigma(N)) \tilde{L}(\tau)+(\sigma(N-2)-\sigma(N)) \tilde{L}^{*}(\tau) .
$$

By the same argument as in the proof of (2.11), we obtain

$$
\begin{aligned}
& \pm i\left[\tilde{H}_{2}(\tau), \sigma\right] \leqq 2 v^{-1}\left\|\sigma_{1}^{\prime}\right\|_{\infty} \frac{1}{2}\left(c_{1}(\tau)+c_{2}(\tau)\right)\left(\varepsilon H_{0}+\left(\bar{b}_{\varepsilon}+1\right) N+2\right) \\
& \quad \leqq\left(\bar{b}_{1}+2\right) v^{-1}\left\|\sigma_{1}^{\prime}\right\|_{\infty}\left(c_{1}(\tau)+c_{2}(\tau)\right)\left(H_{0}+N+1\right) .
\end{aligned}
$$

On the other hand

$$
\begin{aligned}
& \pm i[\tilde{K}(\tau)+\tilde{G}(\tau), R] \\
& \quad \leqq\left\|\left(\mathbb{1}-\frac{1}{2} \Delta\right)^{-1 / 2}\left[k(\tau)+g(\tau), \mathbb{1}-\varrho_{*}\right]\left(\mathbb{1}-\frac{1}{2} \Delta\right)^{-1 / 2}\right\| \|_{1}\left(H_{0}+N\right) \\
& \quad \leqq 4 \gamma_{2} c_{0}(\tau)\left(H_{0}+N\right)
\end{aligned}
$$

by (2.4), (2.5), and (3.9).

Finally, we estimate $\left[\tilde{L}(\tau)+\tilde{L}^{*}(\tau), R\right]$. By the same argument as in the proof of (2.8), we obtain easily

$$
\left[L_{i}^{*}(\tau), R\right]\left[R, L_{i}(\tau)\right] \leqq \frac{1}{2} c_{i}(\tau)^{2} \Gamma_{2}\left((\mathbb{1}-R)\left|V_{i}\right|^{1-\alpha_{i}}(\mathbb{1}-R)\right)
$$

for $i=1,2$. Using (2.10) and momentum variables, we obtain for any $\varepsilon>0$

$$
\begin{aligned}
\Gamma_{2}(\cdot) & \leqq \sum_{i<j}\left(1-\hat{\varrho}\left(k_{i}\right) \hat{\varrho}\left(k_{j}\right)\right)^{2}\left(\frac{\varepsilon}{4}\left(k_{i}-k_{j}\right)^{2}+\bar{b}_{\varepsilon}\right) \\
& \leqq \frac{\varepsilon}{2} N H_{0}+2 \bar{b}_{\varepsilon} N \sum_{i}\left(1-\hat{\varrho}\left(k_{i}\right)\right)^{2} \\
& \leqq \frac{\varepsilon}{2} N H_{0}+2 \bar{b}_{\varepsilon} \gamma_{2}^{2} N\left(H_{0}+N\right) .
\end{aligned}
$$


By the same argument as in the proof of (2.11), (3.14) and (3.15) imply the estimate

$$
\begin{aligned}
\pm & i\left[\tilde{L}(\tau)+\tilde{L}^{*}(\tau), R\right] \leqq\left(\varepsilon^{1 / 2}+2 \varepsilon^{-1 / 2} \bar{b}_{\varepsilon} \gamma_{2}^{2}\right) \\
& \cdot\left(c_{1}(\tau)+c_{2}(\tau)\right)\left(H_{0}+N+1\right) .
\end{aligned}
$$

Collecting (3.12), (3.13), and (3.16) and using Schwarz's inequality, we obtain (3.8). In order to prove the last statement of the lemma, we note that $\gamma_{2} \rightarrow 0$ when $\kappa \rightarrow \infty$ and that the sum $\left(\varepsilon^{1 / 2}+2 \varepsilon^{-1 / 2} \bar{b}_{\varepsilon} \gamma_{2}^{2}\right)$ can be made arbitrarily small by fixing first $\varepsilon$ sufficiently small and then $\kappa$ sufficiently large. Q.E.D.

We next turn to $J_{3}$ and $J_{4}$ in a first step, we obtain the crude estimate

$$
J_{i} \leqq 2 \hbar^{i / 2-1} \int_{s}^{t} d \tau\left\|\Psi_{1}(\tau)\right\|_{+}\left\|\left(H_{0}+N+1\right)^{-1 / 2} \tilde{H}_{i}(\tau) P \Psi_{2}(\tau)\right\|
$$

for $i=3,4$. By part 1) of Condition 2.1, by Lemma 2.2 and Proposition 2.2, the integrand in (3.17) is a continuous function of $\tau$. At the present stage, we can already prove the convergence of $\tilde{W}(t, s)$ to $\tilde{U}_{2}(t, s)$ in finite time intervals.

Proposition 3.1. Assume that $V$ satisfies (3.1), that $\varphi$ satisfies (3.2) and the Eq. (2.9) of [2], and that part 1) of Condition 2.1 holds with $\mathscr{D}_{1} \cap Q\left(N B_{1}\right)$ dense in $\mathscr{H}$. Then

$$
s-\lim _{\hbar \rightarrow 0} \tilde{W}(t, s)=\tilde{U}_{2}(t, s)
$$

uniformly for t, s in compact intervals.

Proof. The proof is analogous to that of Proposition 5.1 of [2]. Q.E.D.

We recall that, for stable potentials in the sense of Condition 2.2, part 1) of Condition 2.1 holds with $\mathscr{D}_{1} \cap Q\left(N B_{1}\right)$ obviously dense in $\mathscr{H}$ (see Proposition 2.4).

We are now left with the task of estimating $J_{3}$ and $J_{4}$ uniformly in time. For this purpose, we need more refined estimates than (3.17). We define the operator $V_{4}$ in $\mathscr{H}_{2}$ by

$$
V_{4}=V\left(\mathbb{1}-\Delta_{\ddagger}\right)^{-1} V
$$

where $\Delta_{\neq}$is the Laplace operator in the difference variable, and the operator $g_{2}(\tau)$ in $\mathscr{H}_{1}$ by

$$
g_{2}(\tau)=\int d y|\varphi(\tau, y)|^{2} V_{y}\left(\mathbb{1}-\frac{1}{2} \Delta\right)^{-1} V_{y},
$$

where $V_{y}$ is the operator of multiplication by $V_{y}(x)=V(x-y)$ in $\mathscr{H}_{1}$.

We also define

$$
\begin{aligned}
& \tilde{V}_{4}(\tau)=\left.U_{0}(\tau)^{*} V_{4} U_{0}(\tau)\right|_{\mathscr{H}_{2}}, \\
& \tilde{g}_{2}(\tau)=u_{0}(\tau)^{*} g_{2}(\tau) u_{0}(\tau),
\end{aligned}
$$

and

$$
\Phi_{i}(\tau)=U_{0}(\tau) \Psi_{i}(\tau) \quad \text { for } \quad i=1,2
$$

We can then estimate $J_{3}$ and $J_{4}$ as follows. 
Lemma 3.3. $J_{3}$ and $J_{4}$ satisfy the estimates

$$
\begin{aligned}
& J_{3} \leqq 2 \hbar^{1 / 2} \int_{s}^{t} d \tau\left\|\Psi_{1}(\tau)\right\|_{+}\left\{\left\langle\Psi_{2}(\tau), P N \Gamma_{1}\left(\tilde{g}_{2}(\tau)\right) P \Psi_{2}(\tau)\right\rangle^{1 / 2}\right. \\
&\left.+\left(\|\nabla \varphi(\tau)\|^{2}+2\|\varphi(\tau)\|^{2}\right)^{1 / 2}\left\langle\Psi_{2}(\tau), P \Gamma_{2}\left(\tilde{V}_{4}(\tau)\right) P \Psi_{2}(\tau)\right\rangle^{1 / 2}\right\} \\
& J_{4} \leqq 2 v^{1 / 2} \hbar \int_{s}^{t} d \tau\left\|\Psi_{1}(\tau)\right\|_{+}\left\langle\Psi_{2}(\tau), P \Gamma_{2}\left(\tilde{V}_{4}(\tau)\right) P \Psi_{2}(\tau)\right\rangle^{1 / 2}
\end{aligned}
$$

Proof. In this proof, we omit the $\tau$ dependence. We consider first $J_{3}$ and estimate successively the contributions of $A_{3}^{*}$ and $A_{3}$ to the scalar product $\left\langle\Psi_{1}, \tilde{H}_{3} P \Psi_{2}\right\rangle$ (cf. (3.23) and (3.24) of [2]).

$$
\begin{aligned}
\left\langle\Psi_{1}, \tilde{A}_{3}^{*} P \Psi_{2}\right\rangle & =\left\langle\Phi_{1}, A_{3}^{*} P \Phi_{2}\right\rangle \\
& =\sum_{N}\left\langle\Phi_{1}, \sum_{i \neq j} V_{i j} \varphi_{i}\left(P \Phi_{2}\right)_{(i)}\right\rangle_{N},
\end{aligned}
$$

where $\langle,\rangle_{N}$ denotes the $N$ particle contribution to the scalar product in $\mathscr{H}$, the subscripts $i$ and $j$ label the variables that occur in $V, \varphi_{i}$ denotes the classical solution taken as function of the variable $i$ and $\left(P \Phi_{2}\right)_{(i)}$ denotes the wave function with variables different from $i$.

The previous scalar product is estimated as

$$
\begin{aligned}
\left|\left\langle\Phi_{1}, A_{3}^{*} P \Phi_{2}\right\rangle\right| \leqq \sum_{N}\left\langle\Phi_{1}, \sum_{i \neq j}\left(\mathbb{1}-\frac{1}{2} \Delta_{j}\right) \Phi_{1}\right\rangle_{N}^{1 / 2} \\
\cdot\left\{\sum_{i \neq j}\left\langle\varphi_{i}\left(P \Phi_{2}\right)_{(i)}, V_{i j}\left(\mathbb{1}-\frac{1}{2} \Delta_{j}\right)^{-1} V_{i j} \varphi_{i}\left(P \Phi_{2}\right)_{(i)}\right\rangle_{N}\right\}^{1 / 2}
\end{aligned}
$$

by Schwarz's inequality and where $\Delta_{j}$ is the Laplace operator in the variable $j$,

$$
\begin{aligned}
\ldots= & \sum_{N}\left\{\left\langle\Phi_{1},\left(H_{0}+N\right) \Phi_{1}\right\rangle_{N}(N-1) \int d y|\varphi(y)|^{2}\right. \\
& \left.\cdot\left\langle P \Phi_{2}, \sum_{j}\left(V_{y}\right)_{j}\left(\mathbb{1}-\frac{1}{2} \Delta_{j}\right)^{-1}\left(V_{y}\right)_{j} P \Phi_{2}\right\rangle_{N-1}\right\}^{1 / 2} \\
= & \sum_{N}\left\langle\Phi_{1},\left(H_{0}+N\right) \Phi_{1}\right\rangle_{N}^{1 / 2}\left\langle P \Phi_{2}, N \Gamma_{1}\left(g_{2}\right) P \Phi_{2}\right\rangle_{N-1}^{1 / 2}
\end{aligned}
$$

by inspection,

$$
\leqq\left\|\Phi_{1}\right\|_{+}\left\langle\Phi_{2}, P N \Gamma_{1}\left(g_{2}\right) P \Phi_{2}\right\rangle^{1 / 2}
$$

by Schwarz's inequality applied to the sum over $N$.

We next consider the contribution of $A_{3}$ to $J_{3}$ :

$$
\begin{aligned}
\left\langle\Psi_{1}, \tilde{A}_{3} P \Psi_{2}\right\rangle & =\left\langle\Phi_{1}, A_{3} P \Phi_{2}\right\rangle \\
& =\sum_{N} \sum_{i \neq j}\left\langle\Phi_{1(i)} \varphi_{i}, V_{i j} P \Phi_{2}\right\rangle_{N} .
\end{aligned}
$$

Therefore

$$
\begin{aligned}
\left|\left\langle\Phi_{1}, A_{3} P \Phi_{2}\right\rangle\right| \leqq & \sum_{N}\left\{\sum_{i \neq j}\left\langle\Phi_{1(i)} \varphi_{i},\left(\mathbb{1}-\Delta_{i j}\right) \varphi_{i} \Phi_{1(i)}\right\rangle_{N}\right\}^{1 / 2} \\
& \cdot\left\{\sum_{i \neq j}\left\langle\Phi_{2}, P V_{i j}\left(\mathbb{1}-\Delta_{i j}\right)^{-1} V_{i j} P \Phi_{2}\right\rangle_{N}\right\}^{1 / 2}
\end{aligned}
$$


by Schwarz's inequality, and where $\Delta_{i j}$ is the Laplace operator in the difference variable $x_{i}-x_{j}$. Now $-\Delta_{i j} \leqq-\frac{1}{2}\left(\Delta_{i}+\Delta_{j}\right)$ and therefore

$$
\begin{aligned}
\sum_{i \neq j}\left\langle\Phi_{1(i)} \varphi_{i},\left(\mathbb{1}-\Delta_{i j}\right) \varphi_{i} \Phi_{1(i)}\right\rangle_{N} \leqq & \|\varphi\|^{2}\left\langle\Phi_{1},\left(H_{0}+N\right) \Phi_{1}\right\rangle_{N-1} \\
& +\frac{1}{2}\|\nabla \varphi\|^{2}\left\langle\Phi_{1}, N \Phi_{1}\right\rangle_{N-1} .
\end{aligned}
$$

On the other hand, the last bracket in the R.H.S. of (3.32) is simply

$$
\{\cdot\}=2\left\langle\Phi_{2}, P \Gamma_{2}\left(V_{4}\right) P \Phi_{2}\right\rangle_{N} .
$$

Substituting (3.33) and (3.34) into (3.32) and applying Schwarz's inequality to the sum over $N$, we obtain

$$
\left|\left\langle\Phi_{1}, A_{3} P \Phi_{2}\right\rangle\right| \leqq\left(\|\nabla \varphi\|^{2}+2\|\varphi\|^{2}\right)^{1 / 2}\left\|\Phi_{1}\right\|_{+}\left\langle\Phi_{2}, P \Gamma_{2}\left(V_{4}\right) P \Phi_{2}\right\rangle^{1 / 2} .
$$

(3.24) follows from (3.30) and (3.35).

We next consider $J_{4}$. We have to estimate

$$
\left\langle\Psi_{1}, \tilde{H}_{4} P \Psi_{2}\right\rangle=\left\langle\Phi_{1}, H_{4} P \Phi_{2}\right\rangle=\sum_{N}\left\langle\Phi_{1}, \sum_{i<j} V_{i j} P \Phi_{2}\right\rangle
$$

By Schwarz's inequality

$$
\begin{aligned}
& \left|\left\langle\Phi_{1}, H_{4} P \Phi_{2}\right\rangle\right| \leqq \sum_{N}\left\{\left\langle\Phi_{1}, \sum_{i<j}\left(\mathbb{1}-\Delta_{i j}\right) \Phi_{1}\right\rangle_{N}^{1 / 2}\right. \\
& \left.\cdot\left\langle\Phi_{2}, P \sum_{i<j} V_{i j}\left(\mathbb{1}-\Delta_{i j}\right)^{-1} V_{i j} P \Phi_{2}\right\rangle_{N}^{1 / 2}\right\} \\
& \leqq\left\langle\Phi_{1},\left(H_{0}+N\right) \Phi_{1}\right\rangle^{1 / 2}\left\langle\Phi_{2}, \frac{1}{2} N P \Gamma_{2}\left(V_{4}\right) P \Phi_{2}\right\rangle^{1 / 2} .
\end{aligned}
$$

From this, (3.25) follows immediately. Q.E.D.

The next step is to estimate the two matrix elements that occur in the R.H.S. of (3.24) and (3.25). Using the definition of $P$ (cf. Sect. 3 of [2]), we obtain

$$
\left\langle\Psi_{2}(\tau), P N \Gamma_{1}\left(\tilde{g}_{2}(\tau)\right) P \Psi_{2}(\tau)\right\rangle \leqq 2 v\left\langle\Psi_{2}(\tau), \Gamma_{1}\left(\tilde{g}_{2 R}(\tau)\right) \Psi_{2}(\tau)\right\rangle,
$$

where

$$
\tilde{g}_{2 R}(\tau)=\varrho_{*} \tilde{g}_{2}(\tau) \varrho_{*} .
$$

The R.H.S. of (3.38) is then estimated exactly in the same way as in [2], (see Lemmas 5.5 and 5.6 of [2]), with the only difference that $g_{2}$ replaces $g_{1}$. We collect the relevant results in the following lemma.

\section{Lemma 3.4}

$$
\begin{aligned}
& \left\langle\Psi_{2}(\tau), \Gamma_{1}\left(\tilde{g}_{2 R}(\tau)\right) \Psi_{2}(\tau)\right\rangle^{1 / 2} \leqq\left\langle\Psi, B_{1} \Psi\right\rangle^{1 / 2}\left\|g_{2}(\tau)^{1 / 2} \varrho_{*} u_{0}(\tau) b_{1}^{-1 / 2}\right\| \|_{1} \\
& \quad+\int_{s}^{\tau} d \tau^{\prime}\left\|(N+1)^{1 / 2} \Psi_{2}\left(\tau^{\prime}\right)\right\|\left\{\left\|g_{2}(\tau)^{1 / 2} \varrho_{*} u_{0}\left(\tau-\tau^{\prime}\right) g\left(\tau^{\prime}\right)\right\| \|_{1}\right. \\
& \left.\quad+2\left\|g_{2}(\tau)^{1 / 2} \varrho_{*} u_{0}\left(\tau-\tau^{\prime}\right) k\left(\tau^{\prime}\right)\right\|_{\mathrm{HS}}\right\} .
\end{aligned}
$$

In order to estimate the second matrix element that occurs in (3.24), (3.25), we fix an interval $I \subset \mathbb{R}$ (possibly unbounded) and choose a constant $c_{3}$ according to 
Corollary 2.1 such that (2.16) holds for all $t$ in $I$. We let then $s, t \in I$ and therefore $\tau \in I$. From the definition of $P$, we obtain easily

$$
P \Gamma_{2}\left(V_{4}\right) P \leqq \gamma_{3}^{2}: \Gamma_{2}\left(V_{4 R}\right)\left(H_{0}+c_{3}(N+1)\right)^{-1}:,
$$

where

$$
V_{4 R}=\left.R V_{4} R\right|_{\mathscr{H}_{2}},
$$

$\gamma_{3}$ is the constant (depending on $v$ and $\kappa$ ) defined by

$$
\gamma_{3}^{2}=\left(\operatorname{Sup}_{N} N \sigma(N+1)^{2}\right)\left(\operatorname{Sup}_{k}\left(c_{3}+\frac{1}{2} k^{2}\right) \varrho(k)^{2}\right)
$$

so that

$$
\gamma_{3}^{2} \leqq v\left(2 c_{3}+\operatorname{Sup}_{k} k^{2} \hat{\varrho}(k)^{2}\right)
$$

and the dots : : denote the normal product. More precisely, $\Gamma_{2}\left(V_{4 R}\right)$ is for each $N$ a sum of two particle operators, and for each term of the sum the operator $\left(H_{0}+c_{3}(N+1)\right)^{-1}$ acts on the $(N-2)$ remaining variables. Using $(2.16)$, we obtain for all $\tau \in I$

$$
\left\langle\Psi_{2}(\tau), P \Gamma_{2}\left(\tilde{V}_{4}(\tau)\right) P \Psi_{2}(\tau)\right\rangle \leqq \frac{3}{2} \gamma_{3}^{2} M_{5}(\tau, \tau)^{2},
$$

where we have introduced the function

$$
M_{5}\left(\tau, \tau^{\prime}\right)=\left\langle\Psi_{2}\left(\tau^{\prime}\right),: \Gamma_{2}\left(\tilde{V}_{4 R}(\tau)\right) \tilde{A}_{2}\left(\tau^{\prime}\right)^{-1}: \Psi_{2}\left(\tau^{\prime}\right)\right\rangle^{1 / 2},
$$

with

$$
\begin{gathered}
\tilde{A}_{2}(\tau)=U_{0}(\tau)^{*} A_{2}(\tau) U_{0}(\tau), \\
\tilde{V}_{4 R}(\tau)=U_{0}(\tau)^{*} V_{4 R} U_{0}(\tau),
\end{gathered}
$$

and

$$
A_{2}(\tau)=H_{2}(\tau)+c_{3}(N+1) .
$$

In order to estimate $M_{5}(\tau, \tau)$, we estimate first $M_{5}(\tau, s)$ and

$$
\dot{M}_{5}\left(\tau, \tau^{\prime}\right) \equiv d M_{5}\left(\tau, \tau^{\prime}\right) / d \tau^{\prime}
$$

Clearly

$$
M_{5}(\tau, s)=\left\langle\Psi,: \Gamma_{2}\left(\tilde{V}_{4 R}(\tau)\right) \tilde{A}_{2}(s)^{-1}: \Psi\right\rangle^{1 / 2} .
$$

This quantity is readily estimated by the following obvious lemma (cf. Lemma 5.7 of [2]):

Lemma 3.5. $M_{5}(\tau, s)$ satisfies the estimate

$$
\begin{aligned}
M_{5}(\tau, s) & \leqq 2^{1 / 2}\left\langle\Psi, \Gamma_{2}\left(\tilde{V}_{4 R}(\tau)\right) \Psi\right\rangle^{1 / 2} \\
& \leqq 2^{1 / 2}\left\langle\Psi, B_{2} \Psi\right\rangle^{1 / 2} \mid\left\|\left(\mathbb{1}-\Delta_{\ddagger}\right)^{-1 / 2} V R U_{0}(\tau) b_{2}^{-1 / 2}\right\|_{2} .
\end{aligned}
$$


We now estimate $\dot{M}_{5}\left(\tau, \tau^{\prime}\right)$. We recall (see the proof of Proposition 2.2) that $A_{2}(\tau)$ satisfies the following inequality, in the sense of quadratic forms on $Q\left(H_{0}+N\right)$ :

$$
\pm\left\{\dot{A}_{2}(\tau)+i\left[H_{2}(\tau), A_{2}(\tau)\right]\right\} \leqq 2 \bar{c}(\tau) A_{2}(\tau)
$$

where $\bar{c}(\tau)$ is defined by $(2.36),(2.27)$, and (2.32).

Lemma 3.6. $\dot{M}_{5}\left(\tau, \tau^{\prime}\right)$ satisfies the estimate

$$
\begin{aligned}
\left|\dot{M}_{5}\left(\tau, \tau^{\prime}\right)\right| \leqq & \bar{c}\left(\tau^{\prime}\right) M_{5}\left(\tau, \tau^{\prime}\right)+\left\|N^{1 / 2} \Psi_{2}\left(\tau^{\prime}\right)\right\| \\
& \cdot\left\{\|\|\left(\mathbb{1}-\Delta_{\neq}\right)^{-1 / 2} V R U_{0}\left(\tau-\tau^{\prime}\right) G\left(\tau^{\prime}\right)\|\|_{2}+4\left\|\mid w_{2}\left(\tau, \tau^{\prime}\right) \varrho_{*}\right\| \|_{1}\right\} \\
& +2^{1 / 2}\|\Psi\|\left\|\left(\mathbb{1}-\Delta_{\neq}\right)^{-1 / 2} V R U_{0}\left(\tau-\tau^{\prime}\right) L^{*}\left(\tau^{\prime}\right) \Psi_{0}\right\|,
\end{aligned}
$$

where $w_{2}\left(\tau, \tau^{\prime}\right) \equiv w_{2}$ is the positive one-particle operator, the square of which has integral kernel

$$
\begin{aligned}
& w_{2}^{2}\left(x_{1}, x_{1}^{\prime}\right)=\int d x_{2} d x_{2}^{\prime} d y V_{4}\left(x_{1}, x_{2} ; x_{1}^{\prime}, x_{2}^{\prime}\right) \\
& \text {. } \overline{\left[\varrho_{*} u_{0}\left(\tau-\tau^{\prime}\right) k\left(\tau^{\prime}\right)\right]\left(x_{2}, y\right)}\left[\varrho_{*} u_{0}\left(\tau-\tau^{\prime}\right) k\left(\tau^{\prime}\right)\right]\left(x_{2}^{\prime}, y\right)
\end{aligned}
$$

with $V_{4}$ defined by (3.19).

Proof. We start from the identity

$$
\begin{aligned}
& 2 i M_{5}\left(\tau, \tau^{\prime}\right) \dot{M}_{5}\left(\tau, \tau^{\prime}\right)=\left\langle\Psi_{2}\left(\tau^{\prime}\right),\left[: \Gamma_{2}\left(\tilde{V}_{4 R}(\tau)\right) \tilde{A}_{2}\left(\tau^{\prime}\right)^{-1}:, \tilde{H}_{2}\left(\tau^{\prime}\right)-H_{0}\right]\right. \\
& \left.\quad+: \Gamma_{2}\left(\tilde{V}_{4 R}(\tau)\right) i \frac{d}{d \tau^{\prime}} \tilde{A}_{2}\left(\tau^{\prime}\right)^{-1}: \Psi_{2}\left(\tau^{\prime}\right)\right\rangle \\
& =-\left\langle\Psi_{2}\left(\tau^{\prime}\right),: \Gamma_{2}\left(\tilde{V}_{4 R}(\tau)\right)\left\{\tilde { A } _ { 2 } ( \tau ^ { \prime } ) ^ { - 1 } \left(i \frac{d}{d \tau^{\prime}} A_{2}\left(\tau^{\prime}\right)\right.\right.\right. \\
& \left.\left.\left.\quad+\left[\tilde{A}_{2}\left(\tau^{\prime}\right), \tilde{H}_{2}\left(\tau^{\prime}\right)-H_{0}\right]\right) \tilde{A}_{2}\left(\tau^{\prime}\right)^{-1}\right\}: \Psi_{2}\left(\tau^{\prime}\right)\right\rangle \\
& \quad+\left\langle\Psi_{2}\left(\tau^{\prime}\right),: \Gamma_{2}\left(\left[\tilde{V}_{4 R}(\tau), \tilde{K}^{\prime}\left(\tau^{\prime}\right)+\tilde{G}\left(\tau^{\prime}\right)\right]\right) \tilde{A}_{2}\left(\tau^{\prime}\right)^{-1}: \Psi_{2}\left(\tau^{\prime}\right)\right\rangle \\
& +\left\langle\Psi_{2}\left(\tau^{\prime}\right),: \Gamma_{2}\left(\tilde{V}_{4 R}(\tau)\right) \tilde{A}_{2}\left(\tau^{\prime}\right)^{-1}:<\tilde{L}^{*}\left(\tau^{\prime}\right) \Psi_{2}\left(\tau^{\prime}\right)\right\rangle \\
& \quad+\left\langle\Psi_{2}\left(\tau^{\prime}\right), \tilde{L}\left(\tau^{\prime}\right)<: \Gamma_{2}\left(\tilde{V}_{4 R}(\tau)\right) \tilde{A}_{2}\left(\tau^{\prime}\right)^{-1}: \Psi_{2}\left(\tau^{\prime}\right)\right\rangle
\end{aligned}
$$

where we have classified the various terms in the commutator with $\tilde{H}_{2}\left(\tau^{\prime}\right)-H_{0}$ according to their connectedness with $\tilde{V}_{4 R}(\tau)$. In particular in the last two terms, the notation $<$ means that $\tilde{L}$ and $\tilde{L}^{*}$ should be contracted with $\tilde{V}_{4 R}$, namely that the operator $\tilde{V}_{4 R}$ acts on one or both particles created by $\tilde{L}^{*}$ or annihilated by $\tilde{L}$ [1]. The two contributions thereby obtained will be referred to as having one or two contractions respectively and will be written with $\frac{1}{1}$ or $\frac{2}{2}$.

Using (3.53), we estimate the first matrix element in (3.57) by

$$
K \cdot\rangle \mid \leqq 2 \bar{c}(\tau) M_{5}\left(\tau, \tau^{\prime}\right)^{2} \text {. }
$$


By Schwarz's inequality, the second matrix element is estimated as

$$
\begin{aligned}
K \cdot\rangle \mid= & 2\left|\operatorname{Im}\left\langle\Psi_{2}\left(\tau^{\prime}\right),: \Gamma_{2}\left(\tilde{V}_{4 R}(\tau)\left(\tilde{K}\left(\tau^{\prime}\right)+\tilde{G}\left(\tau^{\prime}\right)\right)\right) \tilde{A}_{2}\left(\tau^{\prime}\right)^{-1}: \Psi_{2}\left(\tau^{\prime}\right)\right\rangle\right| \\
\leqq & 2 M_{5}\left(\tau, \tau^{\prime}\right)\left\langle\Psi_{2}\left(\tau^{\prime}\right),: \Gamma_{2}\left(\left(\tilde{K}\left(\tau^{\prime}\right)+\tilde{G}\left(\tau^{\prime}\right)\right) \tilde{V}_{4 R}(\tau)\left(\tilde{K}\left(\tau^{\prime}\right)+\tilde{G}\left(\tau^{\prime}\right)\right)\right)\right. \\
& \left.\cdot \tilde{A}_{2}\left(\tau^{\prime}\right)^{-1}: \Psi_{2}\left(\tau^{\prime}\right)\right\rangle^{1 / 2} \\
\leqq & 2 M_{5}\left(\tau, \tau^{\prime}\right)\left|\left\|\left(\mathbb{1}-\Delta_{\ddagger}\right)^{-1 / 2} V R U_{0}\left(\tau-\tau^{\prime}\right)\left(K\left(\tau^{\prime}\right)+G\left(\tau^{\prime}\right)\right) \mid\right\|_{2}\left\|N^{1 / 2} \Psi_{2}\left(\tau^{\prime}\right)\right\|,\right.
\end{aligned}
$$

where we have used the definitions of $\tilde{V}_{4 R}, \tilde{K}$, and $\tilde{G}$ and the inequality (2.16) with $c_{3} \geqq 1$, and the factor $\tilde{A}_{2}\left(\tau^{\prime}\right)^{-1}$ has been used to cancel one power of $N$ coming from $\Gamma_{2}$. By further use of Schwarz's inequality (see the proof of (5.60) in [2]), the contribution of $K$ is estimated by

$$
\|\|\left(\mathbb{1}-\Delta_{\neq}\right)^{-1 / 2} V R U_{0}\left(\tau-\tau^{\prime}\right) K\left(\tau^{\prime}\right)\left|\left\|_{2} \leqq 2\left|\left\|w_{2}\left(\tau, \tau^{\prime}\right) \varrho_{*} \mid\right\|_{1}\right.\right.\right.
$$

with $w_{2}\left(\tau, \tau^{\prime}\right)$ defined by (3.55).

The last two matrix elements in the last member of (3.57) are complex conjugate to each other. We consider the term with $\tilde{L}^{*}$. By Schwarz's inequality, we first obtain for $i=1,2$,

$$
\begin{aligned}
& \left\langle\Psi_{2}\left(\tau^{\prime}\right),: \Gamma_{2}\left(\tilde{V}_{4 R}(\tau)\right) \tilde{A}_{2}\left(\tau^{\prime}\right)^{-1}:-\tilde{L}^{*}\left(\tau^{\prime}\right) \Psi_{2}\left(\tau^{\prime}\right)\right\rangle \mid \\
& \quad \leqq M_{5}\left(\tau, \tau^{\prime}\right)\left\langle\Psi_{2}\left(\tau^{\prime}\right), \tilde{L}\left(\tau^{\prime}\right) \boldsymbol{i}_{i}: \Gamma_{2}\left(\tilde{V}_{4 R}(\tau)\right) \tilde{A}_{2}\left(\tau^{\prime}\right)^{-1}:-\tilde{L}^{*}\left(\tau^{\prime}\right) \Psi_{2}\left(\tau^{\prime}\right)\right\rangle^{1 / 2} .
\end{aligned}
$$

The term with two contractions $(i=2)$ is further estimated by

$$
\begin{aligned}
\ldots & \leqq M_{5}\left(\tau, \tau^{\prime}\right)\left\langle\Psi_{2}\left(\tau^{\prime}\right), \tilde{A}_{2}\left(\tau^{\prime}\right)^{-1} \Psi_{2}\left(\tau^{\prime}\right)\right\rangle^{1 / 2}\left\langle\tilde{L}^{*}\left(\tau^{\prime}\right) \Psi_{0}, \tilde{V}_{4 R}(\tau) \tilde{L}^{*}\left(\tau^{\prime}\right) \Psi_{0}\right\rangle^{1 / 2} \\
& \leqq 2^{1 / 2} M_{5}\left(\tau, \tau^{\prime}\right)\|\Psi\|\left\|\left(\mathbb{1}-\Delta_{\neq}\right)^{-1 / 2} V R U_{0}\left(\tau-\tau^{\prime}\right) L^{*}\left(\tau^{\prime}\right) \Psi_{0}\right\|
\end{aligned}
$$

in a similar way as in (3.59). In order to estimate the term with one contraction $(i=1)$, we first use (2.16) to replace $\tilde{A}_{2}\left(\tau^{\prime}\right)^{-1}$ by $(N+1)^{-1}$, apply once more Schwarz's inequality to the summation over the uncontracted leg of $\tilde{L}^{*}$ and $\tilde{L}$, thereby obtaining

$$
\begin{aligned}
& \leqq 2^{1 / 2} M_{5}\left(\tau, \tau^{\prime}\right)\left\langle\Psi_{2}\left(\tau^{\prime}\right), \tilde{L}\left(\tau^{\prime}\right) \leftarrow: \Gamma_{2}\left(\tilde{V}_{4 R}(\tau)\right)(N+1)^{-1}: \angle \tilde{L}^{*}\left(\tau^{\prime}\right) \Psi_{2}\left(\tau^{\prime}\right)\right\rangle^{1 / 2} \\
& \leqq 2^{1 / 2} M_{5}\left(\tau, \tau^{\prime}\right)\left\langle\Psi_{2}\left(\tau^{\prime}\right), 2 \Gamma_{1}\left(\varrho_{*} w_{2}^{2} \varrho_{*}\right) \Psi_{2}\left(\tau^{\prime}\right)\right\rangle^{1 / 2},
\end{aligned}
$$

where $w_{2}$ is defined by (3.55).

Collecting (3.58)-(3.62), we obtain (3.54). Q.E.D.

Lemma 3.6 provides an estimate of the form

$$
\left|\dot{M}_{5}\left(\tau, \tau^{\prime}\right)\right| \leqq \bar{c}\left(\tau^{\prime}\right) M_{5}\left(\tau, \tau^{\prime}\right)+f\left(\tau, \tau^{\prime}\right)
$$

which we shall use in the integrated form

$$
\begin{aligned}
M_{5}(\tau, \tau) \leqq & M_{5}(\tau, s) \exp \left(\int_{s}^{\tau} d \tau^{\prime} \bar{c}\left(\tau^{\prime}\right)\right) \\
& +\int_{s}^{\tau} d \tau^{\prime} f\left(\tau, \tau^{\prime}\right) \exp \left(\int_{\tau^{\prime}}^{\tau} d \tau^{\prime \prime} \bar{c}\left(\tau^{\prime \prime}\right)\right) .
\end{aligned}
$$

In order to complete the estimation of $J_{3}$ and $J_{4}$, we proceed as in [2], making essential use of the dispersive properties of the free evolution expressed by Lemma 5.9 of [2]. We recall that $V$ and $\varphi$ satisfy the assumptions (3.1) and (3.2), 
and that $b_{i}(i=1,2)$ are defined by (5.3) and (5.4) of [2] with $\beta>1$. We shall write $V=V_{1}+V_{2}$ with $V_{i} \in L^{p_{1}}, i=1,2$. The following two lemmas are the analogues of Lemmas 5.11 and 5.12 of [2].

Lemma 3.7. Let $l>2, l \geqq n / \beta$, and $l \geqq p_{1}$. Define $l_{i}^{\prime}$, $a$ and $\lambda_{l}^{\prime}(\tau)$ by

$$
\begin{aligned}
& 1 / l_{i}^{\prime}=\operatorname{Max}\left(0,1 / p_{i}-1 / l-1 / n\right) \quad \text { for } i=1,2, \\
& a=\operatorname{Sup}_{2 \leqq q \leqq 2 n /(n-2)}\left\|\left(\mathbb{1}-\frac{1}{2} \Delta\right)^{-1 / 2}\right\|_{q \leftarrow 2}\left(\leqq \operatorname{Max}\left(1,2^{1 / 2} a_{n}\right)\right),
\end{aligned}
$$

where $\|\cdot\|_{q \leftarrow q^{\prime}}$ denotes the norm of an operator from $L^{q^{\prime}}$ to $L^{q}$ and $a_{n}$ is the constant in the Sobolev inequality (5.68) of [2], and

$$
\lambda_{l}^{\prime}(\tau)=a a_{l}|\tau|^{-n / l} \sum_{i}\|\varrho\|_{\bar{I}_{i}^{\prime}}\left\|V_{i}\right\|_{p_{i}}
$$

Then

$$
\begin{aligned}
& \|\| g_{2}(\tau)^{1 / 2} \varrho_{*} u_{0}(\tau) b_{1}^{-1 / 2}\|\|_{1} \leqq\|\varphi(\tau)\| \lambda_{l}^{\prime}(\tau), \\
& \left\|\mid\left(\mathbb{1}-\Delta_{\neq}\right)^{-1 / 2} V R U_{0}(\tau) b_{2}^{-1 / 2}\right\| \|_{2} \leqq 2^{-n / l} \lambda_{l}^{\prime}(\tau) .
\end{aligned}
$$

Proof. We first note that for suitably regular $\psi$

$$
\begin{aligned}
\left\|g_{2}(\tau)^{1 / 2} \psi\right\| & =\left\langle\psi, g_{2}(\tau) \psi\right\rangle^{1 / 2} \\
& =\left\{\int d y|\varphi(\tau, y)|^{2}\left\langle\psi, V_{y}\left(\mathbb{1}-\frac{1}{2} \Delta\right)^{-1} V_{y} \psi\right\rangle\right\}^{1 / 2} \\
& \leqq\|\varphi(\tau)\| \operatorname{Sup}_{y}\left\|\left(\mathbb{1}-\frac{1}{2} \Delta\right)^{-1 / 2} V_{y} \psi\right\|
\end{aligned}
$$

From there on, the proof of (3.67) follows by the use of Lemma 5.9 of [2], part 2), of Hölder's and Young's inequalities, and of Sobolev's inequality in the form (3.65).

The proof of (3.68) is first reduced to an estimate of a one-particle operator by the use of Lemma 5.10 of [2], and then proceeds along the same lines. Q.E.D.

Lemma 3.8. Let $l \geqq 2, l \geqq p_{1}$ and $1 / l \geqq 1 / p_{2}-1 / 2$, define $l_{i}^{\prime}$ by (3.64) and $q_{i}^{\prime} b y$

$$
1-2 / q_{i}^{\prime}=1 / p_{i}-1 / l \quad \text { for } \quad i=1,2 \text {, }
$$

and let

$$
\begin{aligned}
\mu_{l}^{\prime}\left(\tau, \tau^{\prime}\right)= & a\left(\sum_{i=1,2}\left\|V_{i}\right\|_{p_{t}}\|\varrho\|_{i_{i}^{\prime}}\right)\left(2 \pi\left|\tau-\tau^{\prime}\right|\right)^{-n / l} \\
& \cdot\left(\sum_{i=1,2}\left\|V_{i}\right\|_{p_{l}}\left\|\varphi\left(\tau^{\prime}\right)\right\|_{q_{i}^{\prime}}^{2}\right) .
\end{aligned}
$$

Then

$$
\begin{aligned}
& \left\|g_{2}(\tau)^{1 / 2} \varrho_{*} u_{0}\left(\tau-\tau^{\prime}\right) g\left(\tau^{\prime}\right)\right\|_{1} \leqq\|\varphi(\tau)\| \mu_{l}^{\prime}\left(\tau, \tau^{\prime}\right), \\
& \left\|g_{2}(\tau)^{1 / 2} \varrho_{*} u_{0}\left(\tau-\tau^{\prime}\right) k\left(\tau^{\prime}\right)\right\|_{\mathrm{HS}} \leqq\|\varphi(\tau)\| \mu_{l}^{\prime}\left(\tau, \tau^{\prime}\right), \\
& \left\|\left(\mathbb{1}-\Delta_{\neq}\right)^{-1 / 2} V R U_{0}\left(\tau-\tau^{\prime}\right) G\left(\tau^{\prime}\right)\right\| \|_{2} \leqq 2 \mu_{l}^{\prime}\left(\tau, \tau^{\prime}\right), \\
& \left\|\left(\mathbb{1}-\Delta_{\neq}\right)^{-1 / 2} V R U_{0}\left(\tau-\tau^{\prime}\right) L^{*}\left(\tau^{\prime}\right) \Psi_{0}\right\| \leqq 2^{-1 / 2-n / l} \mu_{l}^{\prime}\left(\tau, \tau^{\prime}\right), \\
& \|\left(w_{2}\left(\tau, \tau^{\prime}\right) \varrho_{*}\left\|_{1} \leqq\right\| w_{2}\left(\tau, \tau^{\prime}\right) \|_{1} \leqq \mu_{l}^{\prime}\left(\tau, \tau^{\prime}\right),\right.
\end{aligned}
$$

where $w_{2}\left(\tau, \tau^{\prime}\right)$ is defined by (3.55). 
Proof. The proof of (3.72) and (3.73) proceeds as that of (5.79) and (5.81) of [2], with the additional use of (3.69) and Sobolev's inequality in the form (3.65).

In order to prove (3.74), we note that for $i=1,2$,

$$
\begin{aligned}
& \|\|\left(\mathbb{1}-\Delta_{\mp}\right)^{-1 / 2} V_{i} R U_{0}\left(\tau-\tau^{\prime}\right) G\left(\tau^{\prime}\right)\|\|_{2} \\
& \quad \leqq\|\|\left(\mathbb{1}-\Delta_{\neq}\right)^{-1 / 2}\left|V_{i}\right|^{\delta_{i}}\|\|_{2}\left|\left\|\left|V_{i}\right|^{1-\delta_{i}} R U_{0}\left(\tau-\tau^{\prime}\right) G\left(\tau^{\prime}\right) \mid\right\|_{2}\right. \\
& \quad \leqq\left\|( \mathbb { 1 } - \Delta ) ^ { - 1 / 2 } | v _ { i } | ^ { \delta _ { i } } \left|\left\|_{1} 2 \operatorname{Sup}_{y}\right\|\left\|\left|V_{i y}\right|^{1-\delta} \varrho_{*} u_{0}\left(\tau-\tau^{\prime}\right) g\left(\tau^{\prime}\right)\right\| \|_{1}\right.\right.
\end{aligned}
$$

with $\left(1-\delta_{i}\right) / p_{i}=1 / l+1 / l_{i}^{\prime}$, and where $v_{(i)}$ is the operator of multiplication by $V_{(i)}$ in $\mathscr{H}_{1}$.

In the last member of (3.77), the first factor is estimated by the use of Hölder's inequality and Sobolev's inequality in the form (3.65), while the last factor is estimated in the same way as in the proof of (5.80) of [2]. The proof of (3.75) proceeds as that of (5.83) of [2], with an additional use of (3.65).

We finally turn to (3.76). The first inequality is obvious. To prove the second, we write for $\psi \in L^{2}$

$$
\left\|w_{2}\left(\tau, \tau^{\prime}\right) \psi\right\|=\left\{\int d y\left\|\left(\mathbb{1}-\Delta_{\neq}\right)^{-1 / 2} V\left(\psi \otimes \theta_{y}\right)\right\|_{2}^{2}\right\}^{1 / 2},
$$

where

$$
\theta_{y}=\left[\varrho_{*} u_{0}\left(\tau-\tau^{\prime}\right) k\left(\tau^{\prime}\right)\right](\cdot, y)
$$

and $\|\cdot\|_{2}$ denotes the norm in $L^{2}\left(\mathbb{R}^{n} \times \mathbb{R}^{n}\right)$,

$$
\begin{aligned}
\ldots \leqq & \sum_{i=1,2}\left\|(\mathbb{1}-\Delta)^{-1 / 2} v_{i}\right\|_{2 \leftarrow s_{i}^{\prime}} \\
& \cdot\left\{\int d y\left\{\int d \eta\left(\left.\int d \xi\left|\psi\left(\xi+\frac{\eta}{2}\right) \theta_{y}\left(\xi-\frac{\eta}{2}\right)\right|^{2}\right|^{s_{i}^{\prime} / 2}\right]^{2 / s_{i}^{\prime}}\right\}^{1 / 2}\right. \\
= & \sum_{i=1,2}\left\|(\mathbb{1}-\Delta)^{-1 / 2} v_{i}\right\|_{2 \leftarrow s_{i}^{\prime}}\left\{\int d y\left\||\psi|^{2} *\left|\theta_{y}\right|^{2}\right\|_{s_{i}^{\prime} / 2}\right\}^{1 / 2} \\
\leqq & a\|\psi\| \sum_{i=1,2}\left\|V_{i}\right\|_{p_{i}} \gamma_{s_{i}^{\prime}}\left(\tau, \tau^{\prime}\right),
\end{aligned}
$$

where $s_{i}^{\prime}$ is defined by $1 / s_{i}^{\prime}=1 / 2-1 / l-1 / l_{i}^{\prime}$ and $\gamma_{s_{i}^{\prime}}\left(\tau, \tau^{\prime}\right)$ is defined by (5.86) of [2]. Now (3.76) follows from (3.78) and (5.89) of [2]. Q.E.D.

Collecting the estimates contained in Lemmas 3.3-3.8 and neglecting a few numerical factors to simplify the final expression, we obtain

$$
\begin{aligned}
J_{3}+J_{4} \leqq & 2 \int_{s}^{t} d \tau\left\|\Psi_{1}(\tau)\right\|_{+} \operatorname{Inf}_{l} \lambda_{l}^{\prime}(\tau)\left\{(2 v \hbar)^{1 / 2}\|\varphi\|\left\langle\Psi, B_{1} \Psi\right\rangle^{1 / 2}\right. \\
& \left.+3\left(\hbar^{1 / 2}\|\varphi(\tau)\|_{H^{1}}+\hbar v^{1 / 2}\right) \gamma_{3}\left\langle\Psi, B_{2} \Psi\right\rangle^{1 / 2} \exp \left(\left|\int_{s}^{\tau} d \tau^{\prime} \bar{c}\left(\tau^{\prime}\right)\right|\right)\right\} \\
& +6 \int_{s}^{t} d \tau\left\|\Psi_{1}(\tau)\right\|_{+} \int_{s}^{\tau} d \tau^{\prime} \operatorname{Inf}_{l} \mu_{l}^{\prime}\left(\tau, \tau^{\prime}\right)\left\|(N+1)^{1 / 2} \Psi_{2}\left(\tau^{\prime}\right)\right\| \\
& \cdot\left\{(2 v \hbar)^{1 / 2}\|\varphi\|+4\left(\hbar^{1 / 2}\|\varphi(\tau)\|_{H^{1}}+\hbar v^{1 / 2}\right) \gamma_{3} \exp \left(\left|\int_{\tau^{\prime}}^{\tau} d \tau^{\prime \prime} \bar{c}\left(\tau^{\prime \prime}\right)\right|\right)\right\}
\end{aligned}
$$


where we have used the fact that $\|\varphi(\tau)\|=\|\varphi\|$ is actually independent of $\tau,\|\cdot\|_{H^{1}}$ denotes the norm in the Sobolev space $H^{1}$, and the Infimum is taken over the values of $l$ allowed in Lemma 3.7 for $\lambda_{l}^{\prime}$ and in Lemma 3.8 for $\mu_{l}^{\prime}$.

We now state our main result. Since it is an extension of Theorem 5.1 of [2] to the present case, we refer to [2] for the complete statements, and indicate here only the necessary changes in the assumptions.

Theorem 3.1. Let $n \geqq 3$ and let $V$ satisfy

$$
\left\{\begin{array}{l}
V \in L^{p_{1}}+L^{p_{2}} \quad \text { with } \quad \operatorname{Max}\left(\frac{n}{4}, \frac{2 n}{n+2}\right)<p_{2} \leqq p_{1}<\frac{n}{2}, \\
V_{-} \in L^{n / 2}
\end{array}\right.
$$

Let $r$ and $r^{\prime}$ satisfy the (compatible) conditions

$$
\begin{aligned}
& \frac{1}{2}-\frac{1}{n}<\frac{1}{r} \leqq \frac{1}{r^{\prime}}<\frac{1}{2}-\frac{1}{2 n} \\
& \frac{1}{r}<\frac{1}{2}+\frac{1}{n}-\frac{1}{2 p_{2}} .
\end{aligned}
$$

Let $X_{k r}, \mathscr{X}_{a}(\cdot)$ and $\mathscr{X}_{0}(\cdot)$ be defined by (2.1), (2.2), and (2.11) of [2]. Then parts 1) and 2) of Theorem 5.1 of [2] hold.

Let in addition $k \geqq 3$. Then part 3) of Theorem 5.1 of [2] holds, with however $\tilde{W}(t, s)$ and $\tilde{U}_{2}(t, s)$ now satisfying Propositions 2.1 and 2.2 respectively.

Let in addition $k \geqq 3$ and let Condition 2.1 hold with $\mathscr{D}_{1} \cap Q\left(N B_{1}\right)$ dense in $\mathscr{H}$. Then parts 4) and 5) of Theorem 5.1 of [2] hold.

Proof. We refer to the various statements by their numbering in Theorem 5.1 of [2]. The proof is almost identical with that of the latter and we indicate only the necessary changes. Parts 1) and 2) require no change. In order to prove the first statement in part 3), which refers to finite times, it suffices to prove that $\varphi$ satisfies (3.2). This follows immediately from the definition of $\mathscr{X}_{a}(\mathbb{R})$, from the classical Eq. (2.9) of [2] and the condition $k \geqq 3$. The second statement in part 3) is obvious as far as $\tilde{W}(t, s)$ is concerned. In order to prove it for $\tilde{U}_{2}(t, s)$ it is sufficient to prove that in addition to (3.2), $\varphi$ satisfies the assumptions of Proposition 2.3, namely the integrability at $+\infty$ (resp. at $-\infty$, resp. in $\mathbb{R})$ of the quantities $c_{i}(t)$ and $c_{i}^{\prime}(t)$ for $i=0,1,2$, or equivalently the integrability of $\|\varphi(t)\|_{q}^{2}$ and $\|\varphi(t)\|_{q}\|\dot{\varphi}(t)\|_{q}$ for $q=2 \bar{p}_{i}$ and $q=q_{i}, i=1,2$. Since the time decay of $\|\varphi(t)\|_{q}$, as expressed in the definition of $\mathscr{X}_{0}(\mathbb{R})$, improves with increasing $q$, we choose $\alpha_{i}=\operatorname{Min}\left(p_{i}-1,1\right)$ in $(2.7)$ so as to make $q_{i}$ as large as possible. Since $q_{1} \leqq q_{2}$ and $q_{i} \leqq 2 \bar{p}_{i}$, it is sufficient to consider the case $q=q_{1}=\operatorname{Min}\left(2 \bar{p}_{1}, 4\right)$. The previous integrability condition then follows from the time decay of $\|\varphi(t)\|_{q_{1}}$ and $\|\dot{\varphi}(t)\|_{q_{1}}$ which in turn follow from the definition of $\mathscr{X}_{0}(\mathbb{R})$, directly for $\|\varphi(t)\|_{q_{1}}$ and through the use of the Eq. (1.67) of [2] for $\|\dot{\varphi}(t)\|_{q_{1}}$. This condition reduces to $1-2 / q_{1}>1 / n$ and is satisfied since $p_{1}<n$.

The proof of parts 4) and 5) proceeds in the same manner as in Theorem 5.1 of [2]. By Proposition 2.3 and Condition 2.1 respectively, the factors $\left\|(N+1)^{1 / 2} \Psi_{2}\left(\tau^{\prime}\right)\right\|$ and $\left\|\Psi_{1}(\tau)\right\|_{+}$in (3.79) are bounded uniformly in $\hbar$ and uniformly in $\tau, \tau^{\prime}, s$ in the relevant intervals. It is then sufficient to prove the 
integrability at $+\infty$ (resp. at $-\infty$, resp. in $\mathbb{R})$ of $\left\|\varphi\left(\tau^{\prime}\right)\right\|_{q_{i}^{\prime}}^{2}$ for $i=1,2$, with $q_{i}^{\prime} \equiv q_{i}^{\prime}(l)$ defined by (3.70), both for $l=n+\varepsilon$ and $l=n-\varepsilon$ for some $\varepsilon>0$. By the definition of $\mathscr{X}_{0}(\mathbb{R})$, the integrability condition reduces to $1 / p_{i}-1 / l>1 / n$ and is satisfied because $p_{2} \leqq p_{1}<n / 2$. Q.E.D.

\section{References}

1. Friedrichs, K.O.: Perturbation of spectra in Hilbert space. Providence, RI : Am. Math. Soc. 1965

2. Ginibre, J., Velo, G.: The classical field limit of scattering theory for non-relativistic many-boson systems. I. Commun. Math. Phys. 66, 37-76 (1979)

3. Ginibre, J., Velo, G.: On a class of non linear Schrödinger equations with non local interactions. Preprint, 1979

4. Glimm, J., Jaffe, A.: Quantum Field models. In: Statistical mechanics and field theory, pp. 1-108. Les Houches Lectures 1970. De Witt, C., Stora, R. (eds.) London: Gordon and Breach 1971

5. Hepp, K. : Commun. Math. Phys. 35, 265-277 (1974)

6. Kato, T. J. Fac. Sci. Univ. Tokyo, Sect. I, 17, 241-258 (1970)

7. Nelson, E.: J. Funct. Anal. 11, 211-219 (1972)

8. Ruelle, D. : Statistical mechanics. New York: Benjamin 1969

Communicated by A. Jaffe

Received March 5, 1979 